\title{
Calcium dependent plasticity applied to repetitive transcranial magnetic stimulation with a neural field model
}

\author{
M. T. Wilson - P. K. Fung . \\ P. A. Robinson . J. Shemmell . \\ J. N. J. Reynolds
}

Received: date / Accepted: date

\begin{abstract}
The calcium dependent plasticity (CaDP) approach to the modeling of synaptic weight change is applied using a neural field approach to realistic repetitive transcranial magnetic stimulation (rTMS) protocols. A spatially-symmetric nonlinear neural field model consisting of populations of excitatory and inhibitory neurons is used. The plasticity between excitatory cell populations is then evaluated using a $\mathrm{CaDP}$ approach that incorporates metaplasticity. The direction and size of the plasticity (potentiation or depression) depends on both the amplitude of stimulation and duration of the protocol. The breaks in the inhibitory theta-burst stimulation protocol are crucial to ensuring that the stimulation bursts are potentiating in nature. Tuning the parameters of a spike-timing dependent plasticity (STDP) window with a Monte Carlo approach to maximize agreement between STDP predictions and the CaDP results reproduces a realistically-shaped window with two regions of depression in agreement with the existing literature. Developing understanding of how TMS interacts with cells at a network level may be important for future investigation.
\end{abstract}

M. T. Wilson

School of Engineering, Faculty of Science and Engineering, University of Waikato, Private Bag 3105, Hamilton 3240, New Zealand, Tel.: +64-7-8384834, Fax: +64-7-8384835, E-mail: mtwilson@waikato.ac.nz

J. Shemmell

School of Physical Education, Brain Health Research Centre, University of Otago, P.O. Box 56, Dunedin 9054, New Zealand

J. N. J. Reynolds

Department of Anatomy, Brain Health Research Centre, University of Otago School of Medical Sciences, P.O. Box 913, Dunedin 9054, New Zealand

P. K. Fung

Department of Ophthalmology, SUNY Downstate Medical Center, Brooklyn, NY 11203, U.S.A.

P. A. Robinson

School of Physics, University of Sydney, Sydney, NSW 2006, Australia

Center for Integrative Brain Function, University of Sydney, NSW 2006, Australia

Center for Research Excellence, NeuroSleep, 431 Glebe Point Road, Glebe, NSW 2037, Australia 
Keywords Transcranial Magnetic Stimulation • Calcium Dependent Plasticity • Modeling · Theta Burst Stimulation · Neural Field Theory

PACS 87.19.lw $\cdot 87.19 .1 \mathrm{lj} \cdot 87.50 . \mathrm{cf} \cdot 02.70 . \mathrm{Hm}$

\section{Introduction}

The calcium control hypothesis $[7,47,48]$ provides a low-level approach to describing and modeling of activity-induced synaptic weight changes. Here, the key parameter in determining the direction of the change of synaptic weight is the postsynaptic concentration of calcium. It is assumed that calcium is transferred to the postsynaptic cells via NMDA receptors at a rate dependent on postsynaptic voltage. The NMDA receptors are gated by glutamate which is generated by pre-synaptic activity $[47,45,12]$. The model provides a biophysical link between pre- and postsynaptic activity and plasticity. Indeed, calcium dependent plasticity (CaDP) has been widely investigated as a foundation for the observed STDP effects $[46,23,16]$ and allows for the nonlinear addition of spike pairs in complicated spike trains (e.g., spike triplets). A complementary approach is described by metaplasticity, of which the Bienenstock-Cooper-Munro (BCM) theory is an example $[3,4,1]$. Here the direction of synaptic weight change depends on the history of postsynaptic firing rate - if a cell has been firing at a high rate then a higher rate of synaptic input is required to achieve further potentiation of the weight.

Fung et al. have applied CaDP to the modeling of synaptic weight changes within a simple neural field model, both with and without metaplasticity ([13] and [12] respectively). The work developed a link between the microscopic description of plasticity and a larger-scale population based description. A single population of excitatory cells was considered, driven by an external stimulus. Simulations were carried out for paired association stimuli and a set of standard continuous theta-burst stimulation (cTBS) and intermittent theta-burst stimulation (iTBS) protocols for repetitive transcranial magnetic stimulation (rTMS). They were able to reproduce the characteristics of a spike-timing dependent plasticity (STDP) window based on paired association stimuli and predictions for metaplasticity for cTBS and iTBS protocols were in agreement with the range of results found experimentally.

In neural field approaches, average neural firing rates are modeled. These approaches are common and have had considerable success $[24,25,27,5,6,52,53,39$, $38]$. They have been successful at modeling electroencephalogram activity seen in natural sleep [51], alpha and gamma rhythms [40,36], anesthesia [5,62,52], seizures [53] and evoked potentials. A field-based approach to modeling neural activity is reasonable for describing TMS, since a single TMS pulse typically excites a few $\mathrm{cm}^{2}$ of cortex [58]. The field based approach is also particularly appropriate for modelling plasticity, since a cell responds to the integrated effect of many incoming spikes. Assuming around $10^{4}$ synapses per cell and 10 spikes per second per cell, approximately 1000 spikes arrive in the $10 \mathrm{~ms}$ time taken to form one output spike. Individual synaptic strengthening effects can not therefore be distinguished. The field based approach takes a view that the population of spikes is affected by correlations in incoming and outgoing activity. Initially, an STDP approach was implemented to describe plasticity in a neural field scheme [37,11]. Wilson et al. 
applied this to cTBS and iTBS with a two population linear model [60]. However, the CaDP approach has recently been implemented but in a one population neural field model with a restricted range of protocols $[12,13]$.

In rTMS trains of pulses of magnetic field are applied through the scalp to the cortex, with a view to modulating brain connectivity and function. The rapid changes in magnetic field induce an electric field in the brain and therefore electric currents; these in turn can stimulate groups of neurons to fire and invoke lasting changes in function. The mechanisms of rTMS are unclear, and possibly include changes in plasticity, modulation of excitability, and changes in gene expression [32]. Models of rTMS are much needed to uncover the underlying effects. In this paper we focus on the effects of plasticity as a potential mechanism.

rTMS appears to have had some success in treatment of stroke and Parkinson's disease, although results are mixed [35,17,10,54], and may have significance elsewhere such as depression [28,31] and sleep disorders [8].

Continuous theta-burst stimulation involves the repetitive application of groups of pulses (e.g., three pulses at 20 ms separation, repeated five times a second.) Intermittent theta-burst stimulation is similar, but has short stimulation sequences (e.g., $2 \mathrm{~s}$ ) interspersed with periods of no stimulation (e.g., $8 \mathrm{~s}$ ). Generally in the literature, iTBS has been considered as increasing the strength of excitatory-toexcitatory synapses, and cTBS as decreasing their strength, but there is considerable variation in reported results [55], with protocols that are effective in some subjects ineffective (or even having opposite effects) in others [19]. Rothkegel et al. [42] reported that breaks in stimulation are essential for facilitatory effects. However, Funke and Benali suggested that the train length (total application time of rTMS) is a key parameter [14]. Confusion in the literature is also caused by vague or inconsistent use of terms such as 'potentiate' and 'depress', since an increase in synaptic strength of an inhibitory-to-excitatory connection may result in a reduction in electroencephalographic power or evoked response. In this work we use 'potentiate' to mean a strengthening of the synaptic weight - that is a positive (excitatory) weight becomes more positive whereas a negative (inhibitory) weight becomes more negative. We use 'depress' to mean the opposite - synaptic weights move towards zero.

Although some mechanisms are known, the details of the pathways by which rTMS can result in a desired change in plasticity remain largely unclear. It is likely that at high intensity, neural firing activity is induced on excitatory-to-excitatory axons, that lie approximately along the direction of the induced electric field $[14$, $43,50]$. Of specific importance is the gradient of the component of the electric field along these axons $[41,26]$. Excitatory axons are particularly sensitive to this since they can cover more spatial extent than shorter inhibitory axons. The induced firing can in turn excite other circuits, both excitatory and inhibitory, in other brain areas. At lower intensity, TMS pulses are more likely to result in more firing events in inhibitory cells than excitatory cells since the inhibitory cells have lower thresholds for firing $[57,14,22]$. However, the exact details of the pathways remain unclear.

The risk of producing seizures in subjects is not insignificant and this limits the range of protocols that are ethically acceptable on safety grounds [30]. A key experimental problem is the large range of possible protocols. A continuous burst protocol can be parametrized by pulse separation, number of pulses in a burst, burst rate and total protocol length. For an intermittent protocol, one can 
also vary the time for which the bursts are applied (the ON time) and the period between applications (the OFF time). Pulse intensity is also likely to be important. This large parameter space, coupled with the risk of seizure, makes comprehensive and systematic investigation of protocols difficult. Modeling can therefore prove useful in elucidating the various effects, especially when they can be stimulated numerically. Useful models must be reasonable but not overly-complicated, and must complement experiment.

In this work we develop a realistic cortical population-based model that provides a useful tool for the further investigation of rTMS. Specifically, we apply the CaDP modeling approaches to plasticity in a neural field model consisting of a realistic population of both excitatory and inhibitory cortical cells for the first time, including the effects of both short-acting $\mathrm{GABA}_{\mathrm{A}}$ and the longer-acting $\mathrm{GABA}_{\mathrm{B}}$ forms of the inhibitory neurotransmitter. Experimentally, it is known that longlasting inhibitory effects are relevant for determining rTMS effects such as cortical silent period [19]; it is therefore reasonable to include them. We stimulate the model with a wider range of realistic continuous and intermittent TMS protocols than previously discussed in the literature, and consider the results in light of what is known experimentally. In Sec. 2 we summarize the neural field model of Robinson et al. (e.g. $[39,38,61,12])$, as applied to excitatory and inhibitory populations within the cortex, then show how CaDP can be incorporated in this scheme with metaplasticity $[12,13]$. We then describe how the model can be linearized and applied also with STDP [60] which we optimize to give a realistic window function showing regimes of depression and potentiation, in agreement with other literature but using a different method.

Numerical simulations of cTBS and iTBS are carried out for different protocols and intensities. Results are shown in Sec. 3 and discussed in Sec. 4, and conclusions drawn.

\section{Method}

In this section we summarize the modeling approaches and develop underlying theory. We consider first a neural dynamics approach to calcium dependent metaplasticity, then show how this can be interpreted in terms of an STDP window [37]. Full mathematical details are given in the Appendix.

We model the cortex with a population of excitatory $(e)$ cells coupled with a population of inhibitory $(i)$ cells. In order to conveniently include the effect of stimulation, we introduce a third population of excitatory cells $(x)$ which connect to both $e$ and $i$ populations. The model is shown in simplified form in Fig. 1. For the purposes of this work, plasticity can be considered to occur at the excitatoryto-excitatory synapses. However, plasticity between other synaptic populations can also be considered.

\subsection{Modeling}

We now describe the modeling procedure. For simplicity, we leave the mathematical details for the appendix, and avoid duplicating unnecessarily from previous 


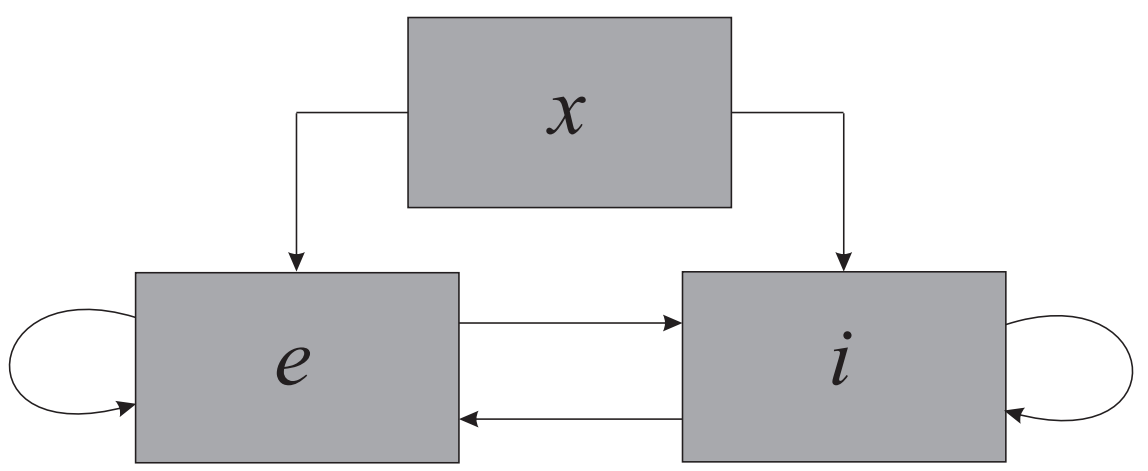

Fig. 1 The neural field model shown in schematic form. Two coupled populations of excitatory $e$ and inhibitory $i$ cells are driven by a third population $x$ of excitatory cells.

work $[37,11,13]$. We first consider the neural field model before developing the description of calcium dependent metaplasticity.

\subsubsection{Neural field model}

We use the well-established neural field model of Robinson et al. [39,38]. This nonlinear model uses the average soma potentials, neural firing rates and axonal pulse rates as its key variables.

We consider the mean soma potential $V_{a}(\mathbf{r}, t)$ of neurons of type $a$ ( $a=e$ or $i$ for excitatory and inhibitory neuronal populations respectively) at a position $\mathbf{r}$ and time $t$. This potential is a result of incoming postsynaptic potentials (PSPs) which are determined by the response of the dendrites to the mean axonal flux rate $\phi_{a b}(\mathbf{r}, t)$, and the synaptic coupling strength between the different populations $\nu_{a b}(\mathbf{r}, t)$. The order of the subscripts denotes the coupling to cells of type $a$ from cells of type $b$. Dendrites are modeled with a differential operator with a Green function equivalent to the impulse response function of the dendrites.

The mean firing rate $Q_{a}(\mathbf{r}, t)$ of population $a$ is a sigmoidal (i.e. nonlinear) function of the mean soma potential. The propagation of mean signals along populations of axons are modeled with a damped wave equation [39].

For simplicity we assume no spatial variation in activity, so the $\mathbf{r}$ label becomes redundant. To some extent this is reasonable since a TMS pulse excites a few $\mathrm{cm}^{2}$ of cortex, but we recognize that spatial effects may be important in human TMS [14] and this remains a topic for future study. We further assume that connections between neurons are randomly distributed, so the synaptic coupling strength $\nu_{a b}(t)$ can be labeled solely by the postsynaptic population, so $\nu_{a b}(t)=\nu_{b}(t)$. Furthermore, we consider the synaptic dynamics as being dominated by the presynaptic cell (that is, the dynamics of the neurotransmitter) implying $\phi_{a b}(t)=\phi_{b}(t)$.

Finally, we add in an external axonal driving stimulation $\phi_{x}$ to both the excitatory and inhibitory populations. We alter the strength of stimulation received by the two different populations $e$ and $i$ by modulating $\phi_{x}$ by parameters $\lambda_{e x}$ and $\lambda_{i x}$, for the $e$ and $i$ destination populations respectively.

The model is provided in full, in both mathematical and diagrammatic form, in the Appendix. Nominal parameter values are also listed there; these are used 
unless stated otherwise. The model and parameter set, without a thalamocortical resonance loop does not produce a strong alpha $(\approx 10 \mathrm{~Hz})$ peak in the spectrum of the firing-rate, but is consistent with the typical 'eyes-open' approach taken in experimental rTMS in order to remove the complicating effects of an alpha rhythm.

\subsubsection{Calcium dependent metaplasticity}

Plasticity can be implemented at a more fundamental level than STDP through consideration of calcium dynamics $[16,49,47,48]$. This was described in detail by Fung and Robinson in [12], with modifications to account for a BCM-type metaplasticity in [13]. CaDP is viewed as a result of coincidence of postsynaptic soma depolarization $V_{a}(t)$ and excitatory glutamate binding, which in turn is a result of presynaptic excitatory activity $\phi_{e}(t)$. When these conditions are obeyed, calcium ions can enter the cell through NMDA receptors and lead to plasticity, the direction of which depends on the calcium level - high levels lead to long-term potentiation (LTP), medium levels to long-term depression (LTD), and low levels to little change [48]. The model we use provides a physiologically-motivated plasticity rule at meso- to macroscopic scales. It contains implicit treatment of the effects of spike times by considering the response of a neuron to be due to the integrated effects of many incoming spikes. Microscale details such as the effects of back-propagating action potentials can be considered implicit in the description through the delayed calcium response to soma depolarization.

Metaplasticity allows for the calcium conductance to vary depending on the history of activity and therefore allows the effective threshold between depression and potentiation to change in a Bienenstock-Cooper-Munro (BCM) type scheme. The result is captured by the ultimate synaptic coupling, (or in a treatment scenario the target synaptic coupling), $\tilde{\nu}_{a b}$. It is this descriptor that is used as the primary indicator of 'plasticity' in this work.

However, there are appreciable delays before this potentiation or depression is realized in practice (e.g. due to protein cascades). The actual synaptic coupling $\nu_{a b}$ at a particular time is a delayed response to $\tilde{\nu}_{a b}$. The full response to a change in $\tilde{\nu}_{a b}$ is therefore not experienced by the system instantaneously, and as a result the actual synaptic coupling $\nu_{a b}$ varies more slowly.

The plasticity modeling evaluates the change in excitatory-to-excitatory synaptic coupling at every time-step of simulation. This coupling $\nu_{e e}$ feeds back into the neural model and changes with time. The model is then advanced forward in time.

\subsection{Application to rTMS}

We now turn our attention to the rTMS protocols. The two cases of cTBS and iTBS offer a range of possibilities since both burst rate (the rate at which bursts are repeated) and number of pulses per burst are variable. Additionally, the amplitude of stimulation becomes a factor. This will make a qualitative difference for CaDP.

Experimentally, amplitudes of TMS are often considered as a percentage of active or resting motor threshold (\%AMT or \%RMT respectively) where threshold is determined as the amplitude such that a motor response is evoked in $50 \%$ of cases. Repetitive TMS is carried out below resting motor threshold. In the case 
of modeling, identifying the exact degree of stimulation corresponding to AMT or RMT is difficult, and is a subject for future work. However, in the nonlinear CaDP approach, the amplitude of stimulation will be important. In terms of the neural models, stimulation can be implemented as a brief increase in external axonal pulse (or spike) rate $\phi_{x}$ over a small time $0.5 \mathrm{~ms}$, which is a typical timescale of a single TMS pulse. This situation corresponds to a stimulation of axons due to the induced electric field in the cortex [41]. In neural field modeling, it is the timeintegrated spike rate that is of significance - this tells us how many spikes occur on average on axons in a given time interval. It is unclear what the experimental measure \%RMT means for the magnitude of the applied pulse rate $\phi_{x}$, since good models of motor evoked potentials are not available. We therefore take a pragmatic view that if a single TMS pulse induces half an extra spike on average on each axon, we will be at motor threshold. Mathematically, this means that we define the time-integrated external pulse rate as $P=\int \phi_{x} d t$ where the integral is over the width of the pulse. A value $P=0.5$ indicates a 'threshold' pulse; smaller values indicate subthreshold.

We emphasize that this is imprecise and is used only to set a plausible amplitude for stimulation in the model. We consider the reasonable results arising from the application of the CaDP model with $P=0.5$ as evidence that this approach is plausible.

The result of the applied stimulation is then given by the following function [60]:

$$
\phi_{x}(t)=\phi_{x}^{\max }\left(\sum_{j} R\left(t-t_{j}^{p}\right)-\left\langle\sum_{j} R\left(t-t_{j}^{P}\right)\right\rangle_{t}\right)
$$

where $t_{j}^{p}$ denotes the time of the $j$-th pulse of the rTMS protocol, $\phi_{x}^{\max }$ is the amplitude of each pulse $(=P / 0.5 \mathrm{~ms}$ where $0.5 \mathrm{~ms}$ is the width of the pulse $)$ and $R(t)$ gives a top-hat function of length $0.5 \mathrm{~ms}$ at $t=0$ :

$$
R(t)=\left\{\begin{array}{l}
1,0<t<0.5 \mathrm{~ms} \\
0, \text { otherwise }
\end{array} .\right.
$$

The subtraction of the mean signal (denoted by the angle brackets) implies that the time average of $\phi_{x}(t)$ over the protocol is zero [60], meaning $\phi_{x}=\delta \phi_{x}$.

A further question concerns the effect of stimulation on the neurons. At high intensity, evidence suggests that axons between excitatory and excitatory neurons are preferentially stimulated $[14,43,50]$, possibly due to their geometry. These long excitatory to excitatory axons, often lying parallel to the cortical surface in a similar direction to the electric field induced by TMS, are susceptible to the changes in gradient of field needed to induce current [41]. At lower intensities (which is more likely to be the case for subthreshold rTMS) the inhibitory cells may activate first since they have a lower threshold for firing $[14,22,57]$ and hence rTMS could be considered as preferentially triggering the inhibitory circuits.

The model allows us to consider both situations by modulating the strength of synaptic couplings $\nu_{e x}$ and $\nu_{i x}$ from the external population to the excitatory and inhibitory populations respectively. A reasonable assumption, based on experiment [57], is that the threshold for stimulation of inhibitory circuits is lower than that for excitatory circuits. Therefore, low intensity pulses predominantly stimulate inhibitory circuits. However, higher intensities, in addition to stimulating the 
inhibitory circuits, strongly stimulate the excitatory to excitatory axons $[50,14]$. Therefore, for the low intensity case, we set parameters $\lambda_{e x}=0.4$ and $\lambda_{i x}=0.6$ where $\lambda_{e x} \nu_{e x}$ and $\lambda_{i x} \nu_{i x}$ denote the strengths of the couplings between the external pulse rate $\phi_{x}$ and the excitatory and inhibitory populations respectively. This gives more stimulation to the inhibitory circuits. For the high intensity case, we set $\lambda_{e x}=1.0$ and $\lambda_{i x}=0.6$, describing a strong excitatory stimulation while maintaining the inhibitory coupling, the latter being above threshold. These values are to some extent arbitrary. We have not investigated the effect of these parameters on the results of this work.

\subsection{Interpretation of model output}

We now comment on how we interpret the output of the model. We start by defining a measure of the plasticity response for a protocol, then we describe how we can use the results to define an equivalent STDP window.

\subsubsection{Quantifying the plasticity response}

For a particular protocol, we use the model to identify the average relative change of target synaptic coupling $\tilde{\nu}_{e e}$ over the first ten seconds of stimulation, normalized per pulse of TMS, $d_{f, p}^{\mathrm{CaDP}, v}$. That is, we define

$$
d_{f, p}^{\mathrm{CaDP}, v}=\frac{1}{M}\left\langle\frac{1}{\tilde{\nu}_{e e}(t=0)} \frac{d \tilde{\nu}_{e e}}{d t}\right\rangle_{t=0 \rightarrow 10 \mathrm{~s}},
$$

where $M$ is the total pulse rate (pulses per burst $p$ multiplied by pulse rate $f$ ), and $v$ can be $C$ or $I$ for the continuous and intermittent protocols respectively. This allows us to identify which protocols are potentiating (in the case of excitatory-toexcitatory synapses, increasing in strength) and which are depressive (in the case of excitatory-to-excitatory synapses, decreasing in strength). This can be done for the cases of large amplitude $(P=0.5$, stimulating the excitatory population with $\left.\lambda_{e x}=1, \lambda_{i x}=0.6\right)$ and a lower amplitude $(P=0.375$, stimulating mostly the inhibitory cells through $\left.\lambda_{e x}=0.4, \lambda_{i x}=0.6\right)$.

\subsubsection{Finding the equivalent STDP window}

Since STDP is a common basis on which to describe plasticity, we seek to find an equivalent STDP window that best matches our results. Our aim is to verify that this window has a sensible shape, thus to some degree showing that the CaDP predictions are reasonable. We emphasize that it is well known that pairwise STDP is not appropriate in some conditions, particularly at high pulse rates. However, given the common use of STDP, it is a reasonable analysis to make. We do this with a Monte-Carlo method based on a least-squares assessment of the similarity between the STDP and CaDP results. We first select a plausible plasticity window function $H_{e e}(\tau)$ and then evaluate the STDP predictions for the initial rate of change of excitatory-to-excitatory synaptic weight for the various cTBS and iTBS protocols with Eq. (30) as described in the Appendix. These predictions are then 
rated against the CaDP predictions with a least-squares method. We define a scalar score-function $S$, for a given STDP function $H_{e e}(\tau)$, by:

$$
S\left[H_{e e}(\tau)\right]=\sum_{f, p}\left(d_{f, p}^{\mathrm{CaDP}, \mathrm{C}}-d_{f, p}^{\mathrm{STDP}, \mathrm{C}}\right)^{2}+\sum_{f, p}\left(d_{f, p}^{\mathrm{CaDP}, \mathrm{I}}-d_{f, p}^{\mathrm{STDP}, \mathrm{I}}\right)^{2}
$$

where the terms $d_{f, p}^{\mathrm{CaDP}, v}$ are given by Eq. (3). The terms $d_{f, p}^{\mathrm{STDP}, \mathrm{C}}$ and $d_{f, p}^{\mathrm{STDP}, \mathrm{I}}$ are the equivalent measures for the STDP predictions for cTBS and iTBS respectively. Explicitly, we have

$$
d_{f, p}^{\mathrm{STDP}, v}=\frac{1}{M} \frac{d w_{e e}}{d t}
$$

where $d w_{e e} / d t$ is given by Eq. (30) for the continuous $(v=C)$ or intermittent $(v=I)$ protocols respectively.

The score function $S$ is a measure of how close the STDP predictions are to CaDP, when considered over the cTBS and iTBS modeled protocols. The Monte Carlo approach takes a trial $H_{e e}(\tau)$, defined at various points $\tau$, modifies is slightly with small random changes at each point $\tau$, and then evaluates a new $S$. If the new $S$ is lower than the original, the modified $H_{e e}(\tau)$ is accepted, if not it is rejected. The process is repeated until $S$ converges. The resulting $H_{e e}(\tau)$ then gives an STDP window function that reproduces the CaDP predictions as well as possible. It is possible that the Monte Carlo simulations result in $S$ being trapped in a local but not global minimum. We have not considered this point in detail, but have repeated the simulations several times from different starting $H_{e e}(\tau)$ and recovered similar functions each time. We explore this further in Section 4 below.

\section{Results}

In order to assess the effects of a wide range of rTMS protocols, we evaluate results as a function of burst rate and pulses per burst. Since the model is nonlinear, we also consider two different intensities of pulses. We start by looking at the case of high-intensity pulses, and then consider lower-intensity stimulation.

We have modeled a range of different rTMS protocols by varying both the number of pulses within a burst (1 to 20), and the burst frequency ( $1 \mathrm{~Hz}$ to $20 \mathrm{~Hz}$, in steps of $1 \mathrm{~Hz}$ ). A pulse rate of $50 \mathrm{~Hz}$ is used, so that successive pulses within a burst are $20 \mathrm{~ms}$ apart. This is done for the cases of both cTBS and iTBS (ON for $2 \mathrm{~s}$, OFF for $8 \mathrm{~s}$ ). Although we use a range for frequencies that goes beyond that traditionally labeled as 'theta' $(4-8 \mathrm{~Hz})$, we use the terminology cTBS and iTBS to denote the continuous and repetitive protocols at these frequencies.

We consider the variation in potentiation and depression across various burst rates and pulses per burst, and find the shape of the STDP window. We then look in more detail at where there is oscillation in synaptic weight, and a case where a very high-firing state occurs that may be related to seizure.

\subsection{High intensity pulses}

We first consider the case of a high amplitude of stimulation. In order to model stimulation of excitatory axons, we have used $P=0.5$ with $\lambda_{e x}=1$ and $\lambda_{i x}=$ 
0.6. This corresponds to a pulse at approximately motor threshold. The cases of cTBS and iTBS have been modeled with various burst frequencies and pulses per burst. Results are shown in Fig. 2. In part (a), the fractional change in target synaptic coupling $\tilde{\nu}_{e e}$ per applied pulse, averaged over the first $10 \mathrm{~s}$ of cTBS stimulation, has been plotted against number of pulses per burst ( $x$-axis) and burst frequency ( $y$-axis). Part (b) shows the equivalent calculation with STDP, optimized through Eq. (4) to be similar to part (a); the optimized $H_{e e}(\tau)$ curve is shown in the inset. Part (c) shows the similar calculation of CaDP with iTBS; the equivalent calculation of STDP is shown in part (d). In the plots the thin dashed line shows the zero-contour, marking the boundary between LTP (yellow/red) and LTD (green/blue). Since the LTD is weak the blue shade is not present on the figure, but it is included on the color scale to give symmetry in the LTP and LTD scale. The solid black line denotes the limit of possibility for protocols above this, one burst will overlap the next burst. One can see that low numbers of pulses per burst tends to favor no change or LTD, whereas higher numbers result in LTP.

In the vicinity of the zero-plasticity contour, there are several protocols for which $\tilde{\nu}_{e e}$ oscillates with time. This implies that a protocol may produce LTD or LTP depending on how long it is run. Protocols for which this occurs are contained within the area marked by the thick dashed line. This is an important prediction of the model and is discussed further in section 3.4 below.

\subsection{Low intensity pulses}

Next we consider the case of lower intensity pulses. Here we have used $P=0.375$ with $\lambda_{e x}=0.4$ and $\lambda_{i x}=0.6$, so mostly inhibitory neurons have been stimulated. Figure 3 shows the results, in a similar form to Fig. 2. Part (a) shows the case of CaDP with cTBS. Plasticity is represented by the fractional change in synaptic weight per pulse of the protocol. The equivalent calculation with STDP is shown in part (b), with the inset giving the $H_{e e}(\tau)$ curve after optimization. Part (c) shows the results of the CaDP calculation with iTBS, and part (d) the equivalent with STDP. The area within the thick dashed lines on the CaDP plots (a) and (c) shows the region over which plasticity is oscillatory. The plots are similar to the equivalent plots of Fig. 2 but the difference between cTBS and iTBS is more pronounced, with the majority of cTBS protocols being depressive and many of the iTBS protocols being strongly potentiating especially at high burst rates with many pulses per burst.

In order to verify consistency in the predictions of the equivalent STDP window, we (a) have carried out the Monte Carlo calculation using two different time windows, and (b) have carried it out many times from different starting points with different random number sequences. Results are shown in Fig. 4 part (a), for the time windows, and (b) for the different simulations.

\subsection{Details of iTBS effects}

To allow some analysis of the differences between cTBS and iTBS protocols, we show the time series results for calcium concentration and target synaptic plasticity 

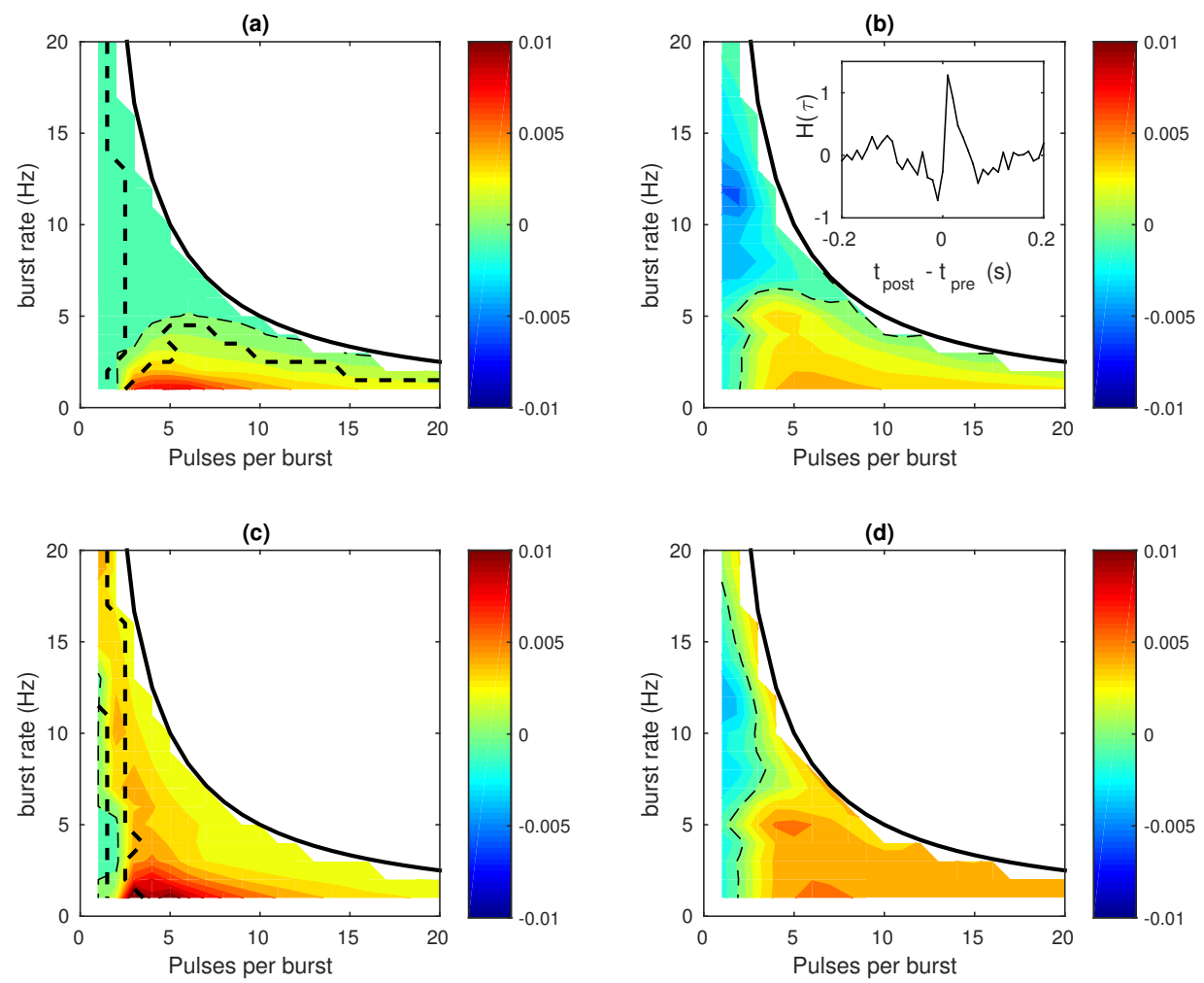

Fig. 2 Color online: A plot of the initial plasticity (average fractional change in $\tilde{\nu}_{e e}$ per TMS pulse) of different rTMS protocols for CaDP and STDP, with a high amplitude of stimulation. (a) Predictions of CaDP for cTBS. The axes denote the number of pulses per burst ( $x$-axis) and the burst frequency ( $y$-axis). The plasticity of an excitatory-to-excitatory synapse is indicated by the color, and shown as the relative (fractional) change in $\tilde{\nu}_{e e}$ averaged per pulse of the TMS protocol over the first $10 \mathrm{~s}$ of the protocol. The thin dotted line marks the boundary between initial facilitation (yellow and red) and depression (green). The area within the thick dashed line, in the vicinity of the zero-contour, denotes a region where the plasticity oscillates in time - i.e. where the end effect (LTP or LTD) is dependent on the length of time of stimulation. (b) Predictions for STDP for cTBS, with details equivalent to (a). Inset: The STDP response function $H_{e e}(\tau)$ that best matches the CaDP responses, shown as the fractional change in synaptic weight as a function of the time difference between a postsynaptic and presynaptic event. (c) Predictions for CaDP for iTBS. (d) Predictions for STDP for iTBS.

for the cases of a cTBS protocol (at $5 \mathrm{~Hz}$ with three $(50 \mathrm{~Hz})$ pulses per burst at high intensity) and an equivalent iTBS protocol with a $2 \mathrm{~s}$ ON time and $8 \mathrm{~s}$ OFF time. Results are shown in Fig. 5. The iTBS protocol results in a net potentiation due to the calcium concentration rising to high levels in the first pulse of a sequence. The cTBS protocol gives depression, since calcium levels remain moderate. We discuss this further in Section 4 below. 

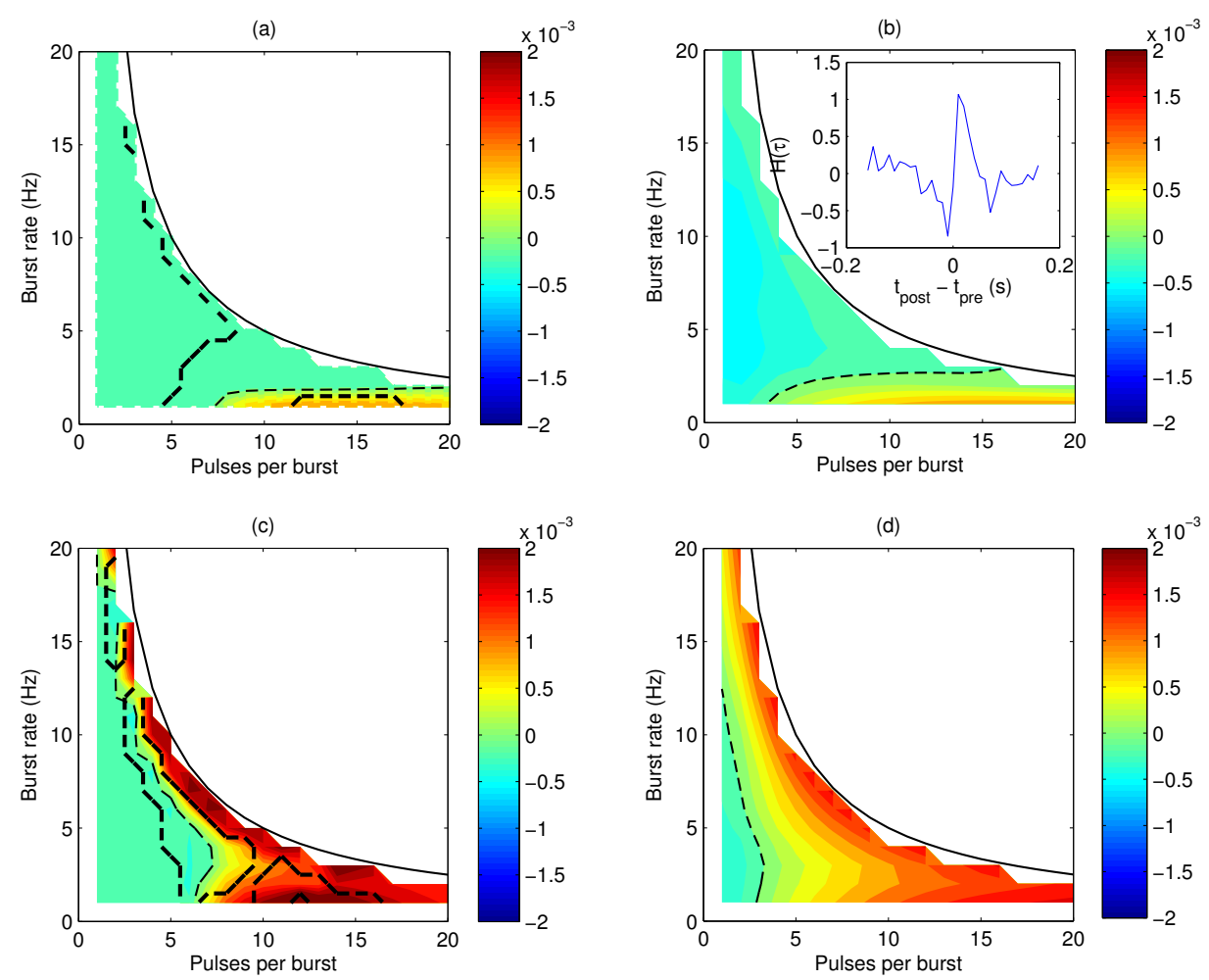

Fig. 3 Color online: A plot of the initial plasticity (average fractional change in $\tilde{\nu}_{e e}$ per TMS pulse) of different rTMS protocols for CaDP and STDP, with a low amplitude of stimulation. Axes are as defined in Fig. 2. (a) Predictions of CaDP for cTBS. (b) Predictions for STDP for cTBS, with details equivalent to (a). Inset: The STDP response function $H_{e e}(\tau)$ that best matches the CaDP responses. (c) Predictions for CaDP for iTBS. (d) Predictions for STDP for iTBS.

\subsection{Oscillations}

To exemplify the situation where there are oscillations, we have chosen one particular protocol (cTBS, high amplitude, 6 pulses per burst, repeated at $6 \mathrm{~Hz}$ ) and shown results in more detail. Figure 6 shows the situation. Part (a) shows the mean soma potential (relative to its equilibrium value) as a function of time, over the first two seconds. The effect of both the individual pulses and the bursts is evident. Part (b) shows the resulting intracellular concentration of calcium. The main plot shows how it changes over $200 \mathrm{~s}$; the inset shows it over a timescale of $2 \mathrm{~s}$. One can see two timescales for oscillation - first, over the burst time of $(0.2 \mathrm{~s})$, second over a much longer timescale, around 1 minute. Part (c) shows how the target synaptic coupling $\tilde{\nu}_{e e}$ changes over this time. The longer timescale is clearly evident here. Finally, part (d) gives the equivalent result in synaptic coupling $\nu_{e e}$ at that time - its response is slower than $\tilde{\nu}_{e e}$ but the oscillations are 
(a)

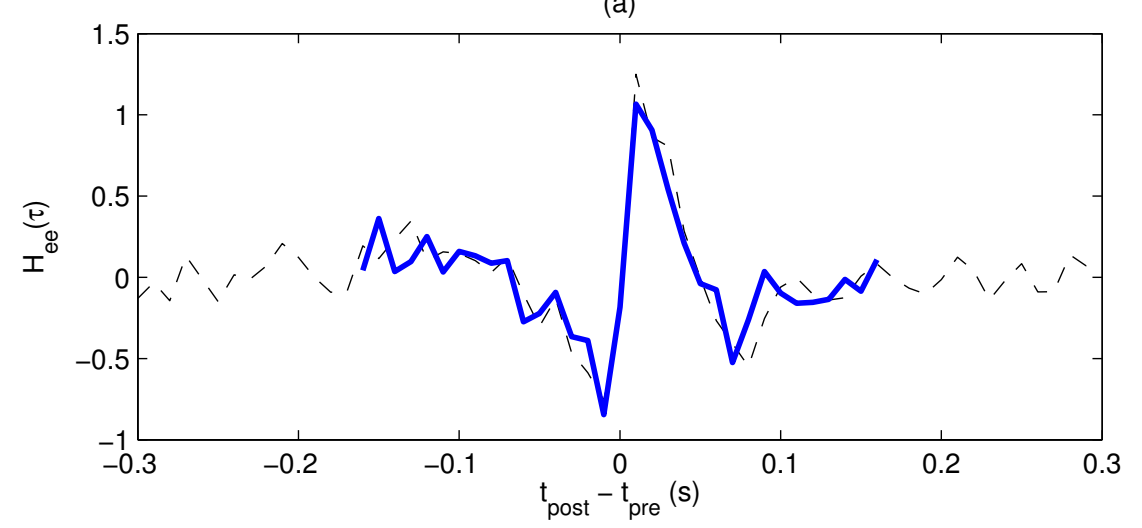

(b)

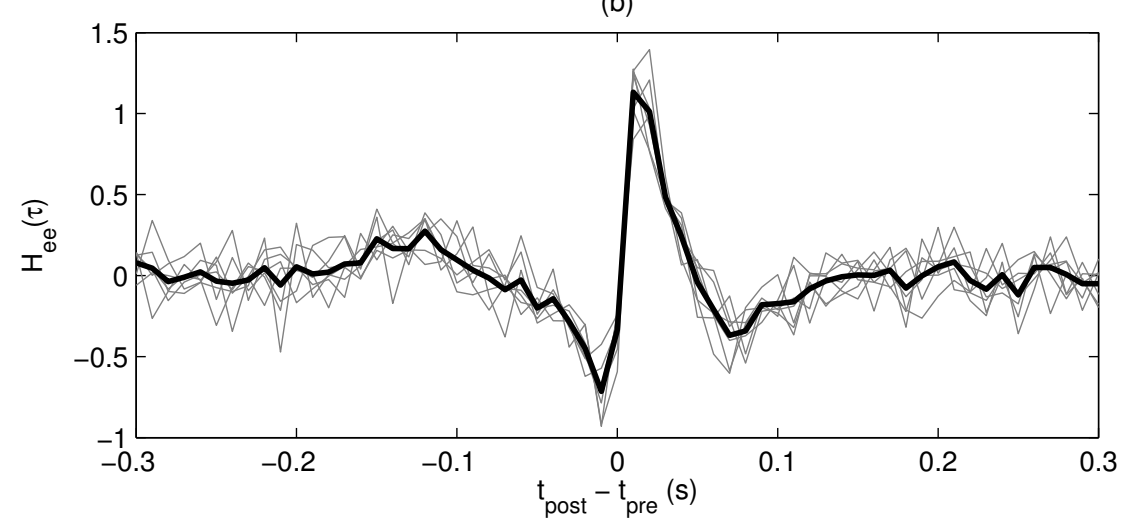

Fig. 4 Color online: (a) The best-fit $H_{e e}(\tau)$ STDP curve for the case of a low intensity pulse as found by a Monte Carlo method over two different domains, $-0.16 \rightarrow 0.16 \mathrm{~s}$ (blue, solid) and $-0.3 \rightarrow 0.3 \mathrm{~s}$ (black, dashed). (b) The results for $H_{e e}(\tau)$ for arising from seven different starting points for the STDP curve (gray lines), and their mean (black line).

clearly evident. Hence, overall, the change in synaptic strength depends on the length of the protocol.

\subsection{High-firing state}

Some highly potentiating protocols, if run for sufficient time, result in a sudden transition in mean firing rate $Q_{a}$ to an unreasonably high value. One might interpret this as a seizure state. Figure 7 shows the situation for an example case (cTBS, high intensity, 6 pulses per burst, repeated at $3 \mathrm{~Hz}$ ). Part (a) shows the mean soma potential. At around $80 \mathrm{~s}$ into the protocol (shown in the inset), the soma potential transitions to an elevated state from which it does not return; 

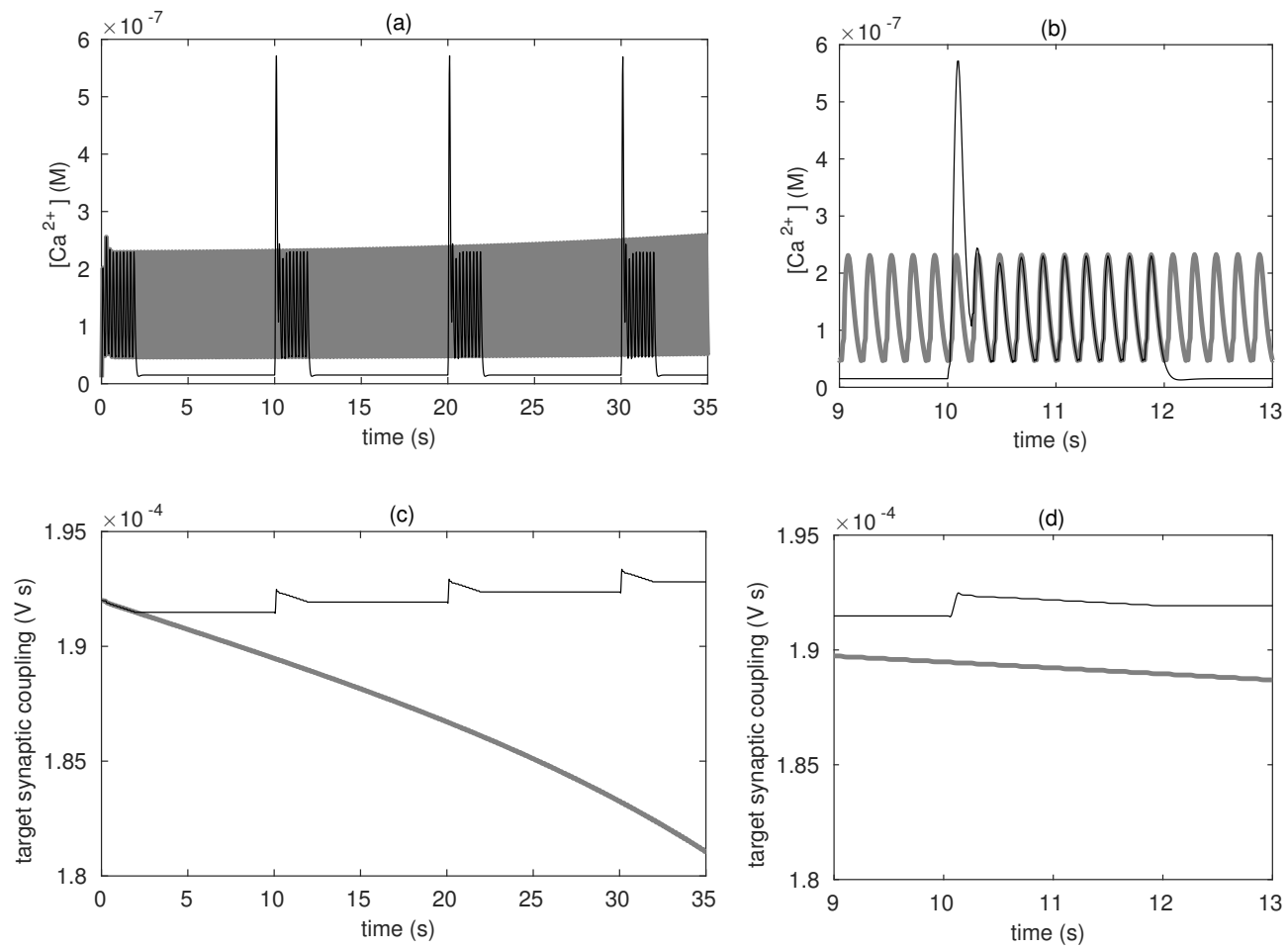

Fig. 5 A plot distinguishing the effects of the cTBS and iTBS protocols. (a) The calcium concentration for cTBS (gray) and iTBS (black) protocols as a function of time. (Three pulses per burst at $50 \mathrm{~Hz}$, at 5 bursts a second. iTBS ON time is $2 \mathrm{~s}$, OFF time is $8 \mathrm{~s}$.) (b) The results of part (a) on an expanded time scale showing the vicinity of the second ON period of the iTBS sequence. (c) The target synaptic coupling as a function of time for cTBS (gray) and iTBS (black). (d) The results of (c) on an expanded time scale in the vicinity of the second ON period of the iTBS sequence.

indeed, the mean soma potential increases with time. The corresponding mean firing rates move from an average of $22 \mathrm{~s}^{-1}$ to close to maximum. This may be representative of a seizure; however, we note that the model has no mechanism by which the seizure can end. Parts (b), (c), and (d) show the equivalent time variation of the intracellular calcium concentration, target synaptic coupling, and synaptic coupling, respectively.

\subsection{Different normalizations}

Figures 2 and 3 show results that have been expressed per pulse of rTMS. This allows us to compare different protocols with equal numbers of pulses, on a roughly equal footing. However, it is possible to look at other normalizations; e.g., per clock time of the protocol or per burst of rTMS. The former is of relevance to the subject or patient - a shorter total treatment time is less unpleasant. The latter may be 

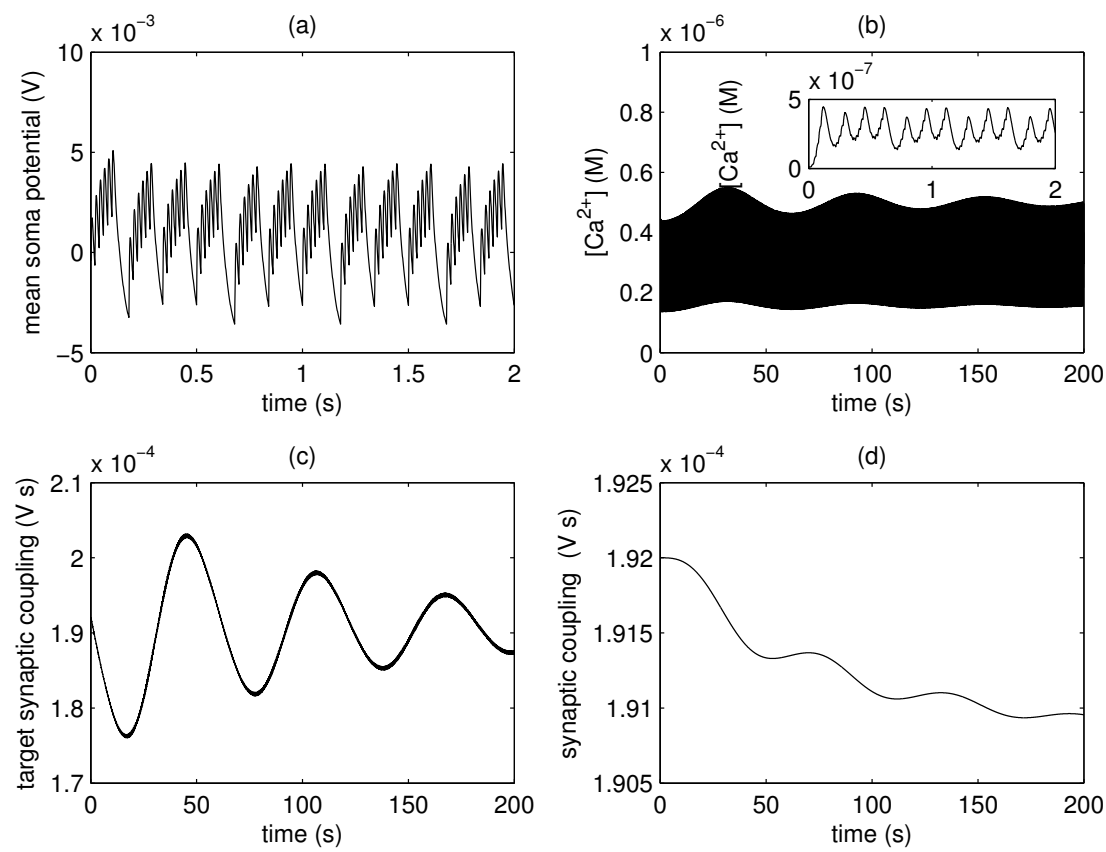

Fig. 6 An example protocol (cTBS, $6 \mathrm{~Hz}, 6$ pulses per burst, high intensity) showing an oscillating plasticity. (a) The mean excitatory soma potential $V_{e}$ as a function of time over the first two seconds. (b) The calcium concentration (moles per litre) over time. Small oscillations are evident over a timescale of order $50 \mathrm{~s}$. The inset shows the same plot over the first two seconds. (c) The target synaptic coupling $\tilde{\nu}_{e e}$ against time, clearly showing oscillations in strength. These oscillations eventually settle. (d) The synaptic coupling $\nu_{e e}$ against time.

particularly useful for interpreting results, since experimentally the time between bursts rather than the number of pulses in a burst is often a key determinant of plasticity. A further normalization would be per energy deposited which would be useful from a safety viewpoint. Assuming that the energy deposited is roughly proportional to the number of pulses times the amplitude of a pulse squared, the shape of the resulting figure would follow that of the per pulse plots of Figs. 2 and 3. To illustrate the different normalization possibilities, Fig. 8(a) and (b) show the results of Fig. 2(a) (the cTBS case) but normalized per second and per burst respectively. We note that in (a) in particular, the potentiating protocols give roughly equivalent potentiation per unit time. The most appropriate choice of normalization for such a plot will depend upon the purpose for which the results are used. 

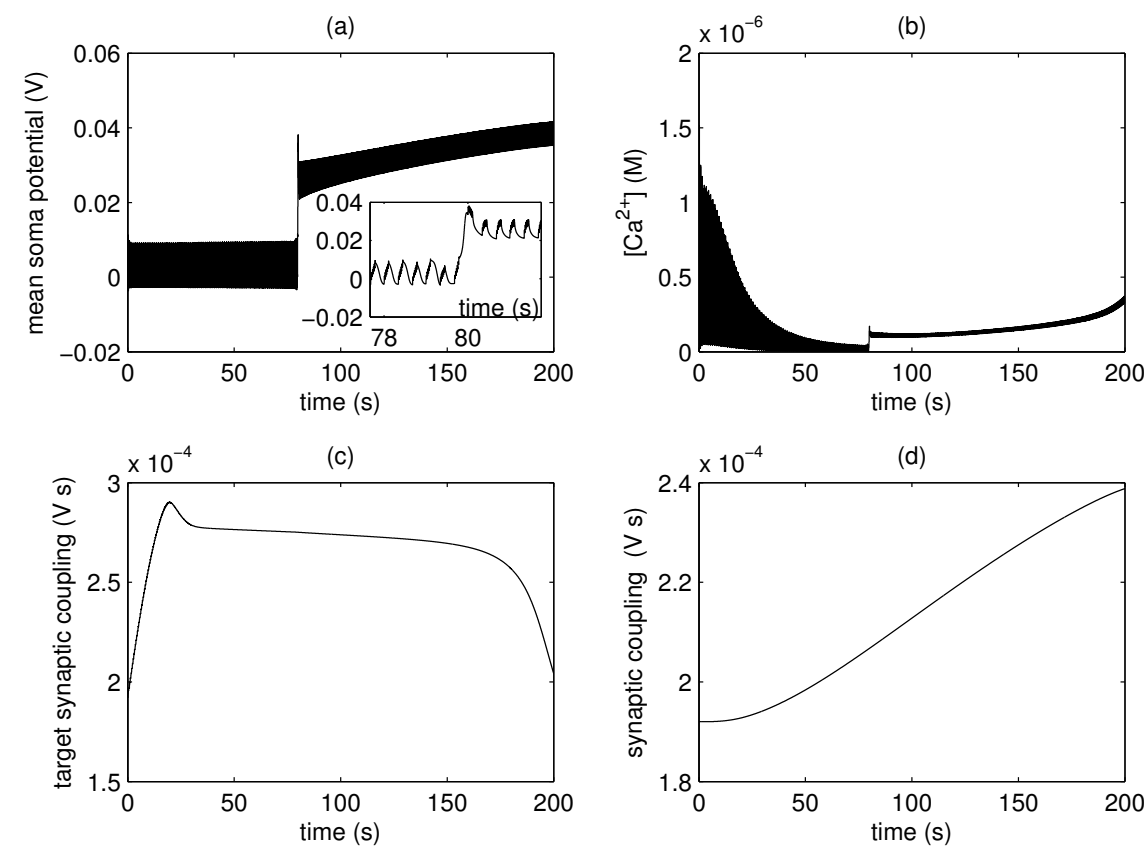

Fig. 7 An example protocol (cTBS, $3 \mathrm{~Hz}, 6$ pulses per burst, high intensity) showing a highfiring rate emerging. (a) The mean excitatory soma potential $V_{e}$ as a function of time. The inset shows the transition to the high-firing state in more detail. (b) The calcium concentration over time. (c) The target synaptic coupling $\tilde{\nu}_{e e}$ against time. (d) The synaptic coupling $\nu_{e e}$ against time.

\section{Discussion}

4.1 Direction of weight change depends on details of the TMS protocol

First, let us consider the general form of the results. In both the high-intensity (Fig. 2) and low-intensity (Fig. 3) cases, protocols can give either potentiation or depression, depending upon the pulses per burst or burst rate and whether the protocol is continuous or intermittent. In some cases, particularly where the initial effect on plasticity is close to zero, plasticity predicted by CaDP can oscillate and the final effect can depend on the length of time for which the protocol is run. Some protocols, especially those that are highly potentiating, can result in a highfiring state when modeled with CaDP. Experimentally, iTBS is widely considered to give rise to LTP and cTBS to LTD [21] but a range of effects are possible [19]. In particular, high frequency $(>5 \mathrm{~Hz})$ stimulation often increases cortical excitability whereas low frequency stimulation $(\sim 1 \mathrm{~Hz})$ lowers it [14]. The model predictions of Fig. 2 and 3 indicate generally strong potentiation for iTBS and either LTP or LTD for cTBS, depending particularly on burst rate. However, the boundary between LTP and LTD depends significantly on the nature of the stimulation (primarily of excitatory axons in Fig. 2 and primarily of inhibitory neurons in Fig. 3), which complicates the comparison. This may explain some of the confusion 
(a)

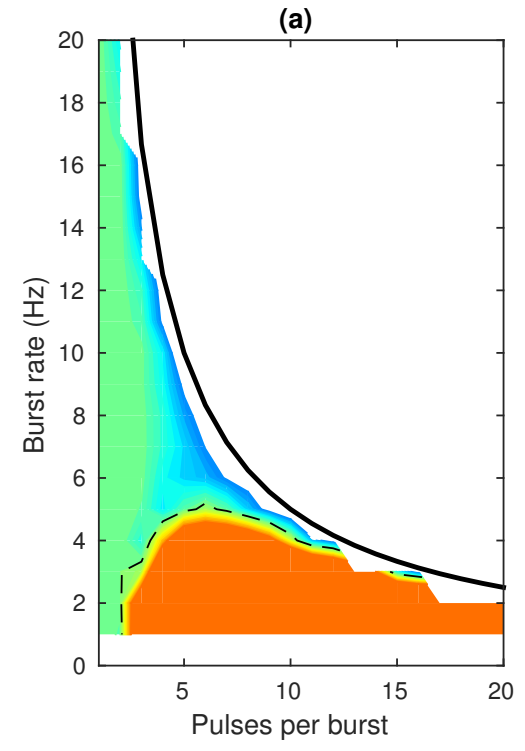

(b)

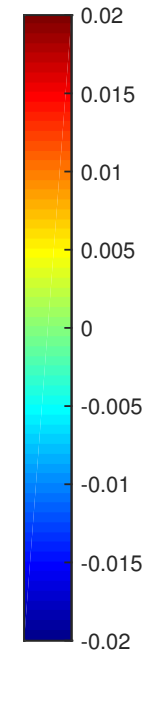

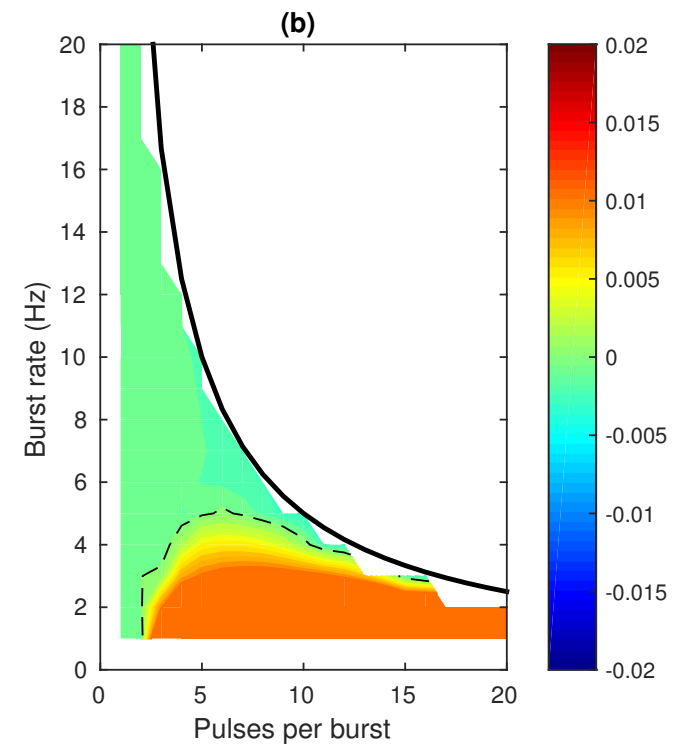

Fig. 8 Color online. (a) A plot of the initial plasticity (average fractional change in $\tilde{\nu}_{e e}$ per second) for different cTBS protocols using CaDP, with a high amplitude of stimulation, normalized per unit time. The units of the color scale are $s^{-1}$. (b) A plot of the initial plasticity normalized per burst (i.e. average fractional change in $\tilde{\nu}_{e e}$ per burst) for the same protocols. The units of the color scale are per burst.

in the literature, where relatively few protocols have been studied in detail. These correspond to isolated points on Figs 2 and 3 and therefore the general form of these plots is largely unexplored experimentally.

\subsection{CaDP applied to rTMS produces a realistic STDP window}

The insets of Fig. 2(b) and Fig. 3(b) show the resulting best-fit STDP $H_{e e}(\tau)$ curves, for the high- and low-intensity stimulation respectively. We note that both curves are quite plausible, with a region of depression when $t_{\text {post }}<t_{\text {pre, }}$, (i.e., the postsynaptic cell fires before the presynaptic cell), but with strong potentiation when $t_{\text {post }}$ is slightly larger than $t_{\text {pre }}$ (i.e., the postsynaptic cell fires very soon after a presynaptic event). In both cases, there is also a small region of LTD when $t_{\text {post }}-t_{\text {pre }}$ is larger (approximately $70-80 \mathrm{~ms}$ ), in agreement with Graupner and Brunel's assessment of the effective STDP window for a paired pulse stimulus with CaDP [16]. The position of this second LTD window is rather later than the positions found experimentally by Nishiyama et al. [29] and Wittenberg \& Wang [63], $20 \mathrm{~ms}$ and $40 \mathrm{~ms}$ respectively. We note that the cell dynamics parameters $\alpha_{a b}$ and $\beta_{a b}$ in particular can affect the position of the window. It is noteworthy that the small amplitude of this second LTD window, in agreement with experiment, arises from an implementation of CaDP based on early theories, e.g., [47,48], as opposed to the more elaborate later theories, e.g., $[49,15]$. The vertical scale of the insets on Fig. 2(b) and Fig. 3(b) is the relative change in synaptic weight per 
postsynaptic pulse, and this is only a little larger in magnitude to the STDP curve produced by $\mathrm{Bi}$ and $\mathrm{Poo}[2]$. The shape of the curve is insensitive to the size of the time domain used to describe $H_{e e}(\tau)$ in the Monte Carlo simulation. In Fig. 4(a) we see, for the low intensity case, the results for $H_{e e}(\tau)$ on two different time domains, specifically $-0.16 \rightarrow 0.16 \mathrm{~s}$ and $-0.30 \rightarrow 0.30 \mathrm{~s}$. The two curves match over the smaller domain, while the results from the larger domain show the function $H_{e e}(\tau)$ decays to zero at both large positive and negative $\tau$, indicating zero plasticity when the pre- and postsynaptic pulses are far apart in time. Moreover, different, starting points for the Monte Carlo simulations recover similar curves. Figure 4(b) shows for the low intensity case seven different results arising from different starting points, in addition to their mean. All produce curves that are similar in shape, consistent with the Monte Carlo simulation being robust to the initial starting point.

This work therefore gives further evidence that a low-level description of plasticity, such as CaDP, leads to the well-known phenomenological STDP description $[23,46,16]$, at least for the protocols considered here. It is known that, at high firing rates, STDP is too simplistic; the effects of successive spikes cannot be considered a superposition of the effects of all the pre- and postsynaptic spike pairs $[46,59,34,9,44]$. Indeed, CaDP simulations (results not shown) of protocols in which pulses are applied $5 \mathrm{~ms}$ apart (as opposed to the $20 \mathrm{~ms}$ for Figs 2 and 3 ) could not be reproduced by the Monte Carlo method using pairwise STDP. Triplet methods such as that described by Pfister and Gerstner [34] can recover and STDP description for high firing rates and map it to a Bienenstock-CooperMunro scheme. Rubin et al. have shown how CaDP can describe STDP triplet results from hippocampal cultures [44]. However, such descriptions are beyond the scope of this paper.

\subsection{Synaptic coupling can oscillate with time}

The CaDP approach to plasticity can predict oscillations in synaptic weight, as exemplified in Fig. 6. Part (c) shows that in this case the target synaptic coupling varies by around $10 \%$ in response to protocols of various lengths. Moreover, the overall response could be either potentiating or depressing, depending upon the length of time for which it is run [14]. This makes comparison on the basis of just burst rate alone, which is common in the early literature, insufficient for interpreting rTMS effects.

\subsection{The OFF time in iTBS is critical for achieving potentiation}

Let us consider the differences between the continuous and intermittent protocols. The two can have markedly different effects, as exemplified in Fig. 2 parts (a) and (c), for cTBS and iTBS respectively. With the cTBS and iTBS protocol of Huang et al. [21], namely 3 pulses per burst repeating at $5 \mathrm{~Hz}$, the cTBS results initially in depression whereas the iTBS ( $2 \mathrm{~s}$ ON, $8 \mathrm{~s}$ OFF) gives an initial potentiation. Figure 5(a) and (b) shows this effect in CaDP through plots of calcium concentration and target synaptic coupling $\tilde{\nu}_{e e}$ respectively for the Huang et al. protocols for cTBS (gray) and iTBS (black). Parts (c) and (d) focus on the time 
between 9 and 13 seconds, which includes $1 \mathrm{~s}$ before and after the second iTBS ON time at $10-12$ seconds.

Here, we see that the first burst of an ON period drives the calcium to a high level. This causes potentiation. Subsequent bursts do not drive calcium to such high concentrations. This medium level of calcium gives an overall depressive effect. The effectiveness of the first burst arises from a lack of inhibition at this time. A stimulating burst drives excitatory cells which then in turn drive the inhibitory population, but with a delay. The calcium concentration can initially rise to high levels before inhibitory responses bring down the overall firing rates and therefore calcium production. The OFF time is therefore crucial to ensure that the network remains highly excitable when receiving its first burst in the ON period of an iTBS sequence. Indeed, Hamada et al. discuss the importance of inhibitory effects for plasticity from an experimental perspective [19].

We emphasize that, per burst, changes in synaptic coupling strength are small; several bursts are required to give a substantial change.

An example of the strong potentiation of this form is seen with quadripulse protocols $[18,20,56]$. In the QPS5 protocol, a burst of four pulses $5 \mathrm{~ms}$ apart was applied once a second [56]. The experimental result gave strong interhemispheric potentiation.

4.5 Amplitude and manner of stimulation affects synaptic coupling changes

The manner of stimulation affects results. The two cases we have looked at are a high-intensity pulse (around motor threshold), and a lower-intensity pulse (subthreshold). It is unclear how to relate quantitatively the machine output as considered by a TMS operator to the amplitude of a pulse within the model. Possibly this could be done by comparing modeled and measured electroencephalographic data following application of many pulses of TMS; the amplitude within the model would be adjusted to give the best fit to the measured data. We have assumed the high-intensity pulse as mostly stimulating the excitatory-to-excitatory axons, whereas the low-intensity pulse preferentially stimulates the inhibitory cells over the excitatory cells. From a modeling perspective, since the spectrum of the resultant activity is dependent greatly on the strength and dynamics of the feedback loops, the manner of stimulation is less important for the determination of qualitative effects. For cTBS, low burst rates and high numbers of pulses per burst give potentiation; for iTBS, low pulses per burst give depression, high pulses per burst leads to potentiation. However, quantitatively, the size of the effect is smaller for the low-intensity stimulation. (Note the difference in the range of the color scale in Figs 2 and 3.)

\subsection{High-firing states can be activated by rTMS}

We note that some protocols appear to be strongly potentiating, particularly with large numbers of pulses per burst. The simulation can move to a high-firing state; an example is shown in Fig. 7. This we interpret as a seizure state - characterized by a sustained high soma potential and consequently high firing. It is well known that there is a risk of seizure with TMS, particularly for strongly potentiating 
protocols [30]. High burst rates and high numbers of pulses per burst are typically not applied experimentally for this reason. However, the potentiating effect of the first burst in a series, e.g., in iTBS or QPS5 [56], suggests that even low burst rates can be highly potentiating and that, if coupled with high stimulus intensities, could induce seizures. We note that in the model there is currently no mechanism by which the seizure can end (e.g., hypoxia); once in this state the simulation does not move from it even when the stimulation is removed. It may be possible to use modeling with appropriate consideration of delay terms $\tau_{a b}$ to predict which protocols are safe to apply experimentally, by quantifying signatures of impending seizures.

\subsection{Assumptions and Limitations}

In this work we have emphasized plasticity as the mechanism for changes in evoked response under rTMS. While this has been the predominant mechanism discussed in literature there is increasing evidence that it cannot be the sole effect - for example evidence for changes in cortical excitability and gene expression have also been discussed [32]. Since experiments to measure plasticity during rTMS are difficult and costly, modeling can play a significant role in describing to what extent calcium-dependent plasticity is capable of describing more easily measured outcomes.

The model used is a field-based model, describing volume averaged effects such as mean firing rates, mean axonal pulse rates, and mean calcium concentration. It cannot quantify changes in individual synapses, but does preserve temporal correlations in these quantities. Thus plasticity effects of spike timings, realized for example through back-propagating action potentials, are implicitly captured. The mathematical details have been presented previously by Robinson [37] for the case of spike-timing dependent plasticity and Fung and Robinson [12] for the case of calcium dependent plasticity.

While many parameters of the model are tightly constrained on biophysical grounds, there are some that are less certain. Notably, the mapping from an experimental stimulation amplitude (e.g. percent resting motor threshold) to values of the external couplings $\lambda_{e x}$ and $\lambda_{i x}$ is uncertain. We have selected values based on the relative extent to which excitatory and inhibitory populations are excited under different amplitude stimulation, but have not investigated how these affect results.

\subsection{Conclusion}

To conclude, we have modeled the plasticity changes due to rTMS using calcium dependent metaplasticity within a two population nonlinear neural field model of brain dynamics. The model allow for variability in intensity and target of stimulation. Changes to synaptic strengths can be positive or negative, depending on the protocols used. They can also depend on the length of time which a protocol is run for. The model also captures the potential for highly potentiating protocols to cause seizure. A realistic STDP window can be generated from the results. The 
role of the iTBS OFF time in producing potentiation through high levels of intracellular calcium has been elucidated. However, we must remark that the modeling has used several simplifying assumptions. In particular, we have not considered the thalamus. The corticothalamic feedback loop is known to be important where frequencies of oscillation are around $10 \mathrm{~Hz}$, due to the resonances between cortex and thalamus $[39,40]$, and is significant in determining whether a cortical system will exhibit a seizure state [37]. This feedback loop will be added in future modeling studies. Additionally, we have not modeled spatial effects explicitly. However, the potential to do this is included in the model, and it is a topic of future work.

Acknowledgements MTW thanks the University of Waikato Research Trust Competitive Fund for financial support. JS was supported by the Neurological Foundation (grant 1128PG) and the University of Otago (research grant 0114-0315). JNJR received support from a Rutherford Discovery Fellowship from the Royal Society of New Zealand. PAR received funding from the Australian Research Council Center of Excellence for Integrative Brain Function (ARC Laureate Fellowship Grant CE140100007) and by ARC Grant FL1401000225.

\section{Appendix}

The neural field model

We use the well-established neural field model of Robinson et al. [39,38]. This model uses the average soma potentials, neural firing rates and axonal pulse rates as its key variables. It is nonlinear since the response of a cell to incoming synaptic events is nonlinear. For completeness, we present the model briefly here, before moving on to consider how synaptic plasticity is incorporated $[12,13]$.

We look at the mean soma potential $V_{a}$ of neurons of type $a(a=e$ or $i$ for excitatory and inhibitory neuronal populations respectively) at a position $\mathbf{r}$ and time $t$. The soma potential is a result of contributions from incoming postsynaptic potentials (PSPs):

$$
V_{a}(\mathbf{r}, t)=\sum_{b} V_{a b}(\mathbf{r}, t)
$$

where $V_{a b}(\mathbf{r}, t)$ is the result of a postsynaptic potential (PSP) of type $b$ onto the cell of type $a(a=e$ or $i ; b=e, i$ or $x)$. The dendritic behavior is governed by

$$
D_{a b} V_{a b}(\mathbf{r}, t)=\nu_{a b}(\mathbf{r}, t) \phi_{a b}\left(\mathbf{r}, t-\tau_{a b}\right),
$$

where the coupling strength $\nu_{a b}(\mathbf{r}, t)$ is the product of the average synaptic weight $s_{a b}(\mathbf{r}, t)$ and the average number of connections $N_{a b}$ to a cell of type $a$ from a cell of type $b$, that is: $\nu_{a b}=s_{a b} N_{a b}$, and $\phi_{a b}$ is the mean axonal pulse rate to a cell of type $a$ from a cell of type $b$. The term $\tau_{a b}$ describes a delay in propagation of the signal to $a$ from $b$ (e.g., introduced by a corticothalamic feedback loop). In general this loop is important for many features of the electroencephalogram especially of order $10-20 \mathrm{~Hz}[39,40]$. However, when a subject has his or her eyes open, as is the case for most TMS studies, the resonance at around $10 \mathrm{~Hz}$ is weak. This allows us to set $\tau_{a b}=0$ for simplicity without losing biophysical relevance. Inclusion of the loop is therefore an important point for future study. In this work we do not consider spatial effects. Therefore we remove reference to the $\mathbf{r}$ label in what follows. To some extent this is reasonable since a TMS pulse excites a few 
$\mathrm{cm}^{2}$ of cortex, but we recognize that spatial effects may be important in human TMS [14] and this remains a topic for future study. The operator $D_{a b}$ describes the time evolution of $V_{a b}$ as a result of synaptic input, and is given by

$$
D_{a b}=\left(\frac{1}{\alpha_{a b}} \frac{d}{d t}+1\right)\left(\frac{1}{\beta_{a b}} \frac{d}{d t}+1\right) .
$$

where $\alpha_{a b}$ and $\beta_{a b}$ describe the combined rates of the synaptic and soma dynamics; specifically $\alpha_{a b}$ is the rise rate of the response of a neuron of type $a$ due to synaptic input from neurons of type $b$ and $\beta_{a b}$ is the decay rate. This is equivalent to the more general propagator form

$$
V_{a b}(t)=\nu_{a b}(t) \int L_{a b}\left(t-t^{\prime}\right) \phi_{a b}\left(t^{\prime}\right) d t^{\prime},
$$

where $L_{a b}$ is the Green function corresponding to $D_{a b}$ with the assumption that $\nu_{a b}$ changes slowly. For this work, we use the differential form of Eq. (8). The average firing-rate of neurons of type $a$ depends on soma potential, with

$$
Q_{a}=Q_{a}^{\max } S\left(V_{a}\right),
$$

where

$$
S\left(V_{a}\right)=\frac{1}{1+e^{-\left(V_{a}-\theta_{a}\right) / \sigma_{a}}} .
$$

To describe propagation of mean signals along populations of axons we model mean axonal pulse rates with the damped wave equation [39]

$$
\mathcal{D}_{a b} \phi_{a b}(t)=Q_{b}(t),
$$

where

$$
\mathcal{D}_{a b}=\left(\frac{1}{\gamma_{a b}} \frac{d}{d t}+1\right)^{2}
$$

Note that Eq. (13) in its full form has a Laplacian term describing spatial variation, but this has been removed since we assume there are no spatial variations. This is equivalent to the integral form:

$$
\phi_{a b}(t)=\iint \Gamma_{a b}\left(t-t^{\prime}\right) Q_{b}\left(t^{\prime}\right) d t^{\prime},
$$

where $\Gamma_{a b}$ is the Green function corresponding to $\mathcal{D}_{a b}, r_{a b}$ denotes the average range of axons to type $a$ cells from type $b$ cells, and $\gamma_{a b}$ is an axonal rate constant. We assume that connections between neurons are randomly distributed, so $\nu_{a b}$ can be labeled solely by the postsynaptic population, so $\nu_{a b}=\nu_{b}$. Furthermore, we consider the synaptic dynamics as being dominated by the presynaptic cell (that is, the dynamics of the neurotransmitter) and so we can also identify that $\gamma_{a b}=\gamma_{b}, \alpha_{a b}=\alpha_{b}, \beta_{a b}=\beta_{b}$, meaning $\phi_{a b}=\phi_{b}$.

We include multiple inhibitory time-scales for the inhibitory synaptic dynamics [33] through both $\mathrm{GABA}_{\mathrm{A}}$ and $\mathrm{GABA}_{\mathrm{B}}$ effects by using two lots of Eq. (7) for the inhibitory cells: $D_{a i}^{\mathrm{A}}$ and $D_{a i}^{\mathrm{B}}$ acting on $V_{a i}^{\mathrm{A}}$ and $V_{a i}^{\mathrm{B}}$ respectively. Their contributions can be added through Eq. (6).

Finally, we add in an external axonal driving stimulation $\phi_{x}$ to both the excitatory and inhibitory populations. We alter the strength of stimulation received 


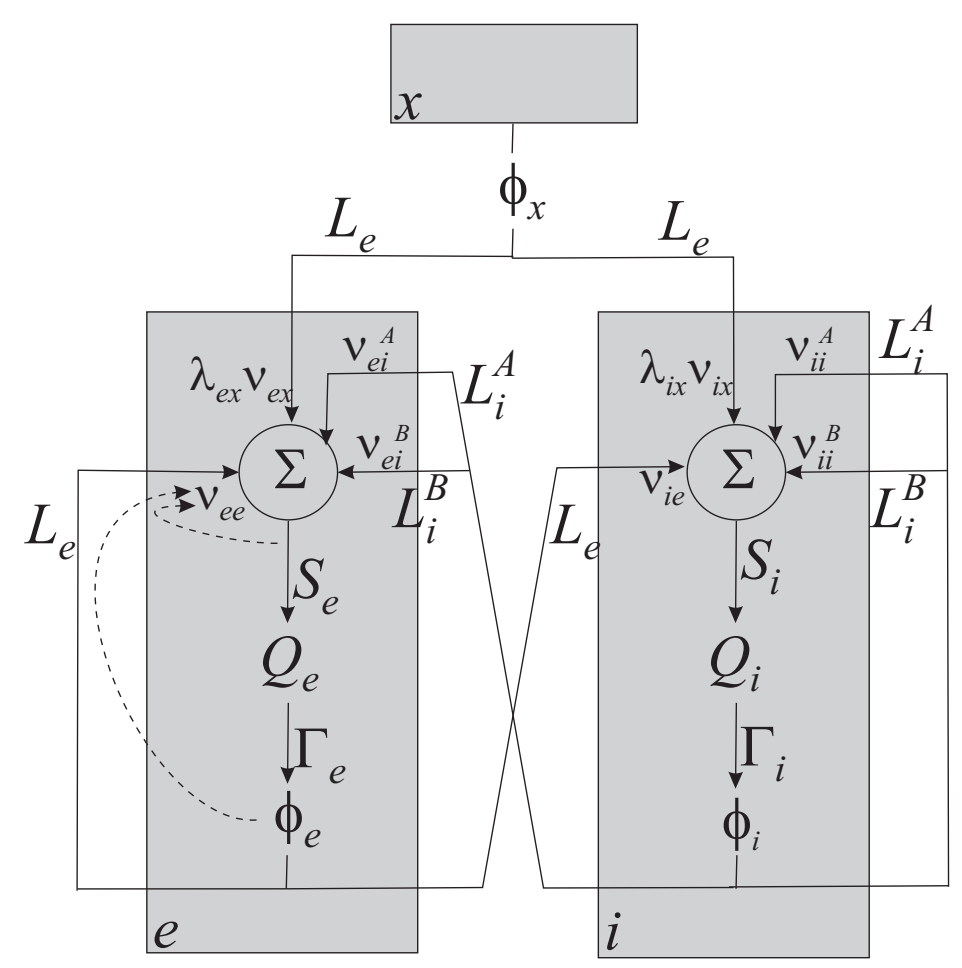

Fig. 9 The populations and connections for the neural field model. Three populations are considered (marked by the gray boxes): an excitatory population $e$, and inhibitory population $i$ and an external excitatory driving population $x$. The dotted lines indicate that the synaptic coupling $\nu_{e e}$ is dependent on $\phi_{e}$ and the excitatory soma potential $V_{e}$ arising from the addition of the PSPs in Eq. (6).

by the two different populations $e$ and $i$ by modulating $\phi_{x}$ by parameters $\lambda_{e x}$ and $\lambda_{i x}$, for the $e$ and $i$ destination populations respectively.

The model is shown in a diagrammatic form in Fig. 9, and the standard parameters for this (taken from references [12] and [13] except for the synaptic coupling terms which have been selected to match the gains used in [60]) are included in Table 1 . The model and parameter set, without a delay term $\tau_{a b}$, cannot produce a strong alpha $(\approx 10 \mathrm{~Hz})$ peak in the spectrum of the firing-rate, but is consistent with the typical 'eyes open' approach taken in experimental rTMS in order to remove the complicating effects of an alpha rhythm.

\section{Calcium dependent metaplasticity}

We now summarize the approach of Fung and Robinson [13] to model the synaptic changes via CaDP with a metaplasticity scheme. The major effector of plasticity is considered to be the postsynaptic intracellular calcium concentration $\left[\mathrm{Ca}^{2+}\right]_{a}$ (where $a$ can represent the excitatory or inhibitory population) which is modulated through NMDA receptors [47]. Specifically, we can model the ultimate synaptic 
Table 1 Parameters for the neural field model and calcium dependent metaplasticity model, drawn from references $[12,13,60]$.

\begin{tabular}{|c|c|c|c|}
\hline Parameter & Description & Value & Unit \\
\hline$\alpha_{e}$ & Cell rise rate to excitatory input & 280 & $\mathrm{~s}^{-1}$ \\
\hline$\beta_{e}$ & Cell fall rate to excitatory input & 70 & $\mathrm{~s}^{-1}$ \\
\hline$\gamma_{e}$ & Excitatory axonal rate constant & 110 & $\mathrm{~s}^{-1}$ \\
\hline$\alpha_{i}^{\mathrm{A}}$ & Cell rise rate to inhibitory $\mathrm{GABA}_{\mathrm{A}}$ input & 400 & $\mathrm{~s}^{-1}$ \\
\hline$\beta_{i}^{\mathrm{A}}$ & Cell fall rate to inhibitory $\mathrm{GABA}_{\mathrm{A}}$ input & 100 & $\mathrm{~s}^{-1}$ \\
\hline$\alpha_{i}^{\mathrm{B}}$ & Cell rise rate to inhibitory $\mathrm{GABA}_{\mathrm{B}}$ input & 20 & $\mathrm{~s}^{-1}$ \\
\hline$\beta_{i}^{\mathrm{B}}$ & Cell fall rate to inhibitory $\mathrm{GABA}_{\mathrm{B}}$ input & 5 & $\mathrm{~s}^{-1}$ \\
\hline$\gamma_{i}$ & Inhibitory axonal rate constant & 1000 & $\mathrm{~s}^{-1}$ \\
\hline$\sigma_{e}$ & $\sqrt{3} / \pi$ times the population st. dev. of the $e$ soma voltages relative to threshold & $3.8 \times 10^{-3}$ & $\mathrm{~V}$ \\
\hline$\theta_{e}$ & Mean threshold for excitatory firing & $13 \times 10^{-3}$ & $\mathrm{~V}$ \\
\hline$\sigma_{i}$ & $\sqrt{3} / \pi$ times the population st. dev. of the $i$ soma voltages relative to threshold & $3.8 \times 10^{-3}$ & $\mathrm{~V}$ \\
\hline$\theta_{i}$ & Mean threshold for inhibitory firing & $13 \times 10^{-3}$ & $\mathrm{~V}$ \\
\hline$Q_{e}^{\max }$ & Maximum excitatory firing rate & 340 & $\mathrm{~s}^{-1}$ \\
\hline$Q_{i}^{\max }$ & Maximum inhibitory firing rate & 340 & $\mathrm{~s}^{-1}$ \\
\hline$\nu_{e e}(t=0)$ & Initial Excitatory-excitatory synaptic coupling & $1.92 \times 10^{-4}$ & $\mathrm{Vs}$ \\
\hline$\nu_{e i}^{\mathrm{A}}$ & $\mathrm{GABA}_{\mathrm{A}}$-modulated inhibitory-excitatory synaptic coupling & $-0.72 \times 10^{-4}$ & $\mathrm{~V} \mathrm{~s}$ \\
\hline$\nu_{e i}^{e i}$ & GABA $_{\mathrm{B}}$-modulated inhibitory-excitatory synaptic coupling & $-0.72 \times 10^{-4}$ & $\mathrm{~V} \mathrm{~s}$ \\
\hline$\nu_{e x}^{e t}$ & External-excitatory synaptic coupling & $1.92 \times 10^{-4}$ & $\mathrm{~V} \mathrm{~s}$ \\
\hline$\nu_{i e}$ & Excitatory-inhibitory synaptic coupling & $1.92 \times 10^{-4}$ & $\mathrm{~V} \mathrm{~s}$ \\
\hline$\nu_{i i}^{\mathrm{A}}$ & GABA $_{A}$-modulated inhibitory-inhibitory synaptic coupling & $-0.72 \times 10^{-4}$ & $\mathrm{~V} \mathrm{~s}$ \\
\hline$\nu_{i i}^{\mathrm{B}}$ & GABA $_{\mathrm{B}}$-modulated inhibitory-inhibitory synaptic coupling & $-0.72 \times 10^{-4}$ & $\mathrm{~V} \mathrm{~s}$ \\
\hline$\nu_{i x}^{i l}$ & External-inhibitory synaptic coupling & $1.92 \times 10^{-4}$ & $\mathrm{~V} \mathrm{~s}$ \\
\hline$\nu_{\max }$ & Maximum synaptic coupling & $10.0 \times 10^{-4}$ & $\mathrm{~V} \mathrm{~s}$ \\
\hline$\lambda_{\text {glu }}$ & Glutamate released per presynaptic excitatory spike & $50 \times 10^{-6}$ & $\mathrm{M} \mathrm{s}$ \\
\hline$g_{a 0}$ & NMDA receptor-modulated calcium conductance at equilibrium & $2 \times 10^{-3}$ & $\mathrm{M} \mathrm{s}^{-1} \mathrm{~V}^{-1}$ \\
\hline$\tau_{\mathrm{Ca}}$ & Time-constant for calcium dynamics & $50 \times 10^{-3}$ & $\mathrm{~s}$ \\
\hline$\tau_{\text {glu }}$ & Time-constant for glutamate dynamics & $30 \times 10^{-3}$ & $\mathrm{~s}$ \\
\hline$\tau_{\text {rec }}$ & Timescale for recovery of calcium conductance & 1000 & $\mathrm{~s}$ \\
\hline$\tau_{\mathrm{BCM}}$ & Timescale for metaplasticity & 7 & $\mathrm{~s}$ \\
\hline$z_{e e}$ & Characteristic time delay in transduction of synaptic strength & 100 & $\mathrm{~s}$ \\
\hline
\end{tabular}

weight $\tilde{s}_{a b}(t)$ through

$$
\frac{d \tilde{s}_{a b}}{d t}=\eta\left(\left[\mathrm{Ca}^{2+}\right]_{a}\right)\left(s_{\max } \Omega\left(\left[\mathrm{Ca}^{2+}\right]_{a}\right)-\tilde{s}_{a b}\right) .
$$

The ultimate synaptic weight $\tilde{s}_{a b}$ describes the value that the synaptic weight will ultimately come to if all external stimulation is stopped - that is, it determines ultimately whether a protocol will show LTP or LTD. However, the actual synaptic weight responds slower than this, as explained below. Equation (15) has a Green function that represents an exponential decay of $s_{a b}$ towards $s_{\max } \Omega$ with a rate parameter $\eta$. Both $\eta$ and $\Omega$ depend on postsynaptic concentration of $\mathrm{Ca}^{2+}$; the rate parameter $\eta$ is low at low $\mathrm{Ca}^{2+}$ concentrations and high at moderate to high concentrations; whereas $\Omega$ is approximately 0.5 at low concentrations $(<0.15 \mu \mathrm{M})$, 0 at medium concentrations $(0.15-0.5 \mu \mathrm{M})$, and rises to 1 at high concentrations $(>0.5 \mu \mathrm{M})$, representing little plasticity, LTD, and LTP respectively.

The actual synaptic weight $s_{a b}$ will respond more slowly, for example due to a sequence of protein cascades. We model this through a differential equation

$$
\left(z_{a b} \frac{d}{d t}+1\right)^{2} s_{a b}=\tilde{s}_{a b} .
$$


where $z_{a b}$ is a characteristic response timescale.

The postsynaptic calcium concentration $\left[\mathrm{Ca}^{2+}\right]_{a}$ (where $a$ can represent an excitatory or inhibitory cell) itself depends on the glutamate binding and postsynaptic activity through

$$
\frac{d\left[\mathrm{Ca}^{2+}\right]_{a}}{d t}=g_{a} B([\mathrm{glu}]) H\left(V_{a}\right)-\frac{\left[\mathrm{Ca}^{2+}\right]_{a}}{\tau_{\mathrm{Ca}}},
$$

where $g_{a}$ is the NMDA receptor-modulated calcium permeability, $B$ models the extent of glutamate binding through a sigmoid function of glutamate concentration [glu], $H$ represents a voltage-dependent modulation of the dynamics (increasing with $V_{a}$ except at very high depolarizations), and $\tau_{\mathrm{Ca}}$ a time-constant for calcium dynamics.

Metaplasticity is incorporated in a Bienenstock-Cooper-Munro (BCM) scheme [4]. In the BCM approach, the activity level that demarcates the boundary between LTD and LTP is dependent on previous activity. This can be incorporated into the CaDP plasticity approach by making the calcium conductance $g_{a}$ dependent upon the history of the weight $s_{a b}$. Specifically, we can write

$$
\frac{d g_{a}}{d t}=\frac{1}{\tau_{\text {rec }}}\left(g_{a 0}-g_{a}\right)-\frac{g_{a 0}}{\tau_{\mathrm{BCM}}}\left(\frac{\tilde{s}_{a b}}{s_{a b}}-1\right)
$$

where $g_{a 0}$ represents the NMDA receptor-modulated calcium conductance at equilibrium, $s_{a b}$ is the actual synaptic weight, and $\tilde{s}_{a b}$ is the ultimate synaptic weight (termed 'target synaptic weight' by Fung et al. [13]). There are two timescales here; $\tau_{\mathrm{BCM}}$ describes the timescale of the metaplasticity and $\tau_{\mathrm{rec}}$, which is much longer than $\tau_{\mathrm{BCM}}$, is the timescale for calcium conductance to recover to the stable value.

Finally, the glutamate concentration depends on generation by presynaptic activity, with a decay over a timescale $\tau_{\text {glu }}$, as modeled by the equation

$$
\frac{d[\mathrm{glu}]}{d t}=\lambda_{\mathrm{glu}} \sum_{E} \phi_{E}-\frac{[\mathrm{glu}]}{\tau_{\mathrm{glu}}},
$$

where $\lambda_{\text {glu }}$ is the glutamate concentration released per presynaptic excitatory spike and $\sum_{E} \phi_{E}$ is the incoming synaptic flux summed over all excitatory populations.

Equations (15) - (19), with associated functions $\eta, \Omega, B$ and $H$ [12], represent CaDP with metaplasticity. The resulting $s_{a b}$ is fed back into Eq. (7) through $\nu_{a b}=N_{a b} s_{a b}$. Equivalently, we can define Eqs. (15), (16) and (18) in terms of the couple $\nu_{a b}$, through $\tilde{\nu}_{a b}=N_{a b} \tilde{s}_{a b}$ and $\nu_{\max }=N_{a b} s_{\max }$ which is what is done in this work. In this way, we can work with the synaptic couplings $\tilde{\nu}_{a b}$ and $\nu_{a b}$ rather than the $\tilde{s}_{a b}, s_{a b}$ and $N_{a b}$.

Parameters and functions for these equations are as defined in [12] and [13]; parameters are listed in Table 1.

Deriving an equivalent STDP window

In this section we describe how an equivalent STDP calculation can be carried out assuming a window function $H_{e e}(\tau)$. To do this we first linearize the model then transform to Fourier space. This allows us to find the spectra of fluctuations in 
firing rate $\delta Q_{a}(t)$ and fluctuation in axonal pulse rate $\delta \phi_{a b}(t)$ in response to the external stimulus $\phi_{x}(t)$.

Since the neural field model is mostly linear, with Eq. (11) and changes due to plasticity being the only nonlinear contributions, linearizing is straightforward. We can write

$$
Q_{a}(t)=Q_{a}^{\mathrm{eqm}}+\delta Q_{a}(t)
$$

and

$$
\phi_{a b}(t)=\phi_{a b}^{\mathrm{eqm}}+\delta \phi_{a b}(t)
$$

where $Q_{a}^{\mathrm{eqm}}$ and $\phi_{a b}^{\mathrm{eqm}}$ are the equilibrium cell firing rates and mean axonal pulse rates, respectively.

We then use the Fourier form in time. We use the following definition of the Fourier representation of a periodic function $x(t)$ with period $T$ :

$$
x(t)=\sum_{n} x_{n} e^{-i \omega_{n} t}
$$

where $\omega_{n}=2 \pi n / T$ with $n$ an integer, so that

$$
x_{n}=\frac{1}{T} \int_{0}^{T} x(t) e^{i \omega_{n} t} d t .
$$

This is a useful form to use since we are considering rTMS, where the stimulation is repetitive over a time-scale $T$. We can then consider $x(t)$ to repeat in a time-scale $T$, this is reasonable if $T$ is much smaller than the timescale over which weights change. We note that for iTBS, $T$ will be typically $10 \mathrm{~s}(2 \mathrm{~s}$ ON followed by $8 \mathrm{~s}$ $\mathrm{OFF}$ ), whereas the mean weights may vary over a scale of order one minute or longer $[11,60,35]$ so such a description is reasonable. The function $x(t)$ and its Fourier representation $x_{n}$ carry the same dimensions.

We can write the Eqs (7) and (12) as

$$
\delta Q_{a n}=T \sum_{b} G_{a b} L_{a b n} \delta \phi_{a b n}
$$

and

$$
\delta \phi_{a b n}=T \Gamma_{a b n} \delta Q_{b n}
$$

where

$$
\begin{gathered}
L_{a b n}=\frac{1}{T\left(1-i \omega_{n} / \alpha_{a b}\right)\left(1-i \omega_{n} / \beta_{a b}\right)}, \\
\Gamma_{a b n}=\frac{1}{T\left(1-i \omega_{n} / \gamma_{a b}\right)^{2}},
\end{gathered}
$$

and

$$
G_{a b}(t)=\left.\nu_{a b}(t) \frac{\partial Q_{a}}{\partial V_{a b}}\right|_{\text {eqm }}
$$

is the gain, which we have assumed to vary only slowly with time.

In Eq. (24), the sum over the suffix $b$, denoting the presynaptic population, is taken over the excitatory population $e$ and both $\mathrm{GABA}_{\mathrm{A}}$ and $\mathrm{GABA}_{\mathrm{B}}$ effects of the inhibitory population $i$, as shown in the ' $\Sigma$ ' signs of Fig. 9. 
Equations (24)-(28) are now simply linear and algebraic. They can be solved with matrix techniques for a given stimulation protocol $[37,60]$.

We now incorporate an STDP description of plasticity into the linearized model. Robinson [37] and Fung et al. [11] showed how this can be done, and again for completeness we summarize it here. We define an STDP window function $H_{a b}(\tau)$, meaning that a postsynaptic event a time $\tau$ after a presynaptic event (for a $b$-to- $a$ synapse) gives a relative change in synaptic weight of $H_{a b}(\tau)$.

We now define a relative synaptic weight $w_{a b}$ such that $w_{a b}=\nu_{a b}(t) / \nu_{a b}(t=$ $0)$, so that $w_{a b}(t=0)=1$. The rate of change of relative synaptic weight is then given by $[37,11]$

$$
\frac{d w_{a b}}{d t}=\int_{-T / 2}^{T / 2}\left\langle\delta Q_{a}\left(t^{\prime}+\tau\right) H_{a b}(\tau) \delta \phi_{a b}\left(t^{\prime}\right)\right\rangle_{t^{\prime}} d \tau
$$

where the temporal average denoted by the angle brackets is centered on $t$ and both the integral and the temporal average are taken over a timescale long enough that $H_{a b}(\tau)$ is negligible for $\tau>T / 2$. The assumption here is that the STDP is pairwise since the integral of Eq. (29) implicitly considers all pairs of presynaptic and postsynaptic events equally, but does not include any consideration of higherorder (e.g., triplet) terms [46].

In Fourier space, Eq. (29) can be written as a straightforward sum over all frequencies [37], making its evaluation fast, with

$$
\frac{d w_{a b}}{d t}=T \sum_{n} \delta Q_{a n}^{*} \delta \phi_{a b n} H_{a b n} .
$$

The spectra $\delta Q_{a n}$ and $\delta \phi_{a n}$ are found for a particular protocol. Then, for a given $H_{a b}(\tau)$, we can Fourier transform to find $H_{a b n}$ and use the sum in Eq. (30) to quickly find the rate of change of weight as predicted by pairwise STDP. Although it is quick to evaluate Eq. (30), if the change in synaptic weight is fed back into the calculation through the excitatory-to-excitatory gain, calculations must be performed over discrete time steps so some of the efficiency is lost. However, since the weights change much more slowly than the firing rates and mean axonal pulse rates, we can use large time steps for updating the synaptic weight. In this work, we concentrate on the initial rate of change of synaptic weight and therefore do not feed the weight back into the calculation.

\section{References}

1. Abraham, W.C., Bear, M.F.: Metaplasticity: the plasticity of synaptic plasticity. TRENDS in Neurosciences 19, 126-130 (1996)

2. Bi, G.Q., Poo, M.M.: Synaptic modifications in cultured hippocampal neurons: dependence on spike timing, synaptic strength, and postsynaptic cell type. Journal of Neuroscience 18, 10,464-10,472 (1998)

3. Bienenstock, E., Lehmann, D.: Regulated criticality in the brain? Advances in complex systems 1, $361-384(1998)$

4. Bienenstock, E.L., Cooper, L.N., Munro, P.W.: Theory for the development of neuron selectivity: orientation specificity and binocular interation in visual cortex. Journal of Neuroscience 2, $32-48$ (1982)

5. Bojak, I., Liley, D.T.J.: Modelling the effects of anaesthesia on the electroencephalogram. Physical Review E 71, 41,902 (2005) 
6. Bojak, I., Liley, D.T.J.: Axonal velocity distributions in neural field equations. PLoS Computational Biology 6, e1000,653 (2010)

7. Castellani, G.C., Quinlan, E.M., Cooper, L.N., Yeung, L.C., Shouval, H.Z.: A biophysical model of bidirectional synaptic plasticity: dependence on AMPA and NMDA receptors. Proceedings of the National Academy of Sciences 98, 12,772-12,777 (2001)

8. Civardi, C., Collini, A., Monaco, F., Cantello, R.: Applications of transcranial magnetic stimulation in sleep medicine. Sleep Medicine Reviews 13, 35-46 (2009)

9. Clopath, C., Gerstner, W.: Voltage and spike timing interact in STDP - a unified model. Frontiers in Synaptic Neuroscience 2, 25 (2010)

10. Fregni, F., Simon, D.K., Wu, A., Pascual-Leone, A.: Biophysical mechanisms of multistability in resting-state cortical rhythms. Journal of Neurology, Neurosurgery and Psychiatry 76, 1614-1623 (2005)

11. Fung, P.K., Haber, A.L., Robinson, P.A.: Neural field theory of plasticity in the cerebral cortex. Journal of Theoretical Biology 318, 44-57 (2013)

12. Fung, P.K., Robinson, P.A.: Neural field theory of calcium dependent plasticity with applications to transcranial magnetic stimulation. Journal of Theoretical Biology 324, 72-83 (2013)

13. Fung, P.K., Robinson, P.A.: Neural field theory of synaptic metaplasticity with applications to theta burst stimulation. Journal of Theoretical Biology 340, 164-176 (2014)

14. Funke, K., Benali, A.: Cortical cellular actions of transcranial magnetic stimulation. Restorative Neurology and Neuroscience 28, 399-417 (2010)

15. Graupner, M., Brunel, N.: STDP in a bistable synapse model based on CaMKII and associated signalling pathways. PLoS Computational Biology 3, 2299-2323 (2007)

16. Graupner, M., Brunel, N.: Mechanisms of induction and maintenance of spike-timing dependent plasticity in biological synapse models. Frontiers in Computational Neuroscience 4, 1-19 (2010)

17. Hallett, M.: Transcranial magnetic stimulation: A primer. Neuron 55, 187-199 (2007)

18. Hamada, M., Hanajima, R., Terao, Y., Arai, N., Furubayashi, T., Inomata-Terada, S., Yugeta, A., Matsumoto, H., Shirota, Y., Ugawa, Y.: Quadro-pulse stimulation is more effective than paired pulse stimulation for plasticity induction of the human motor cortex. Clinical Neurophysiology 118, 2672-2682 (2007)

19. Hamada, M., Murase, N., Hasan, A., Balaratnam, M., Rothwell, J.C.: The role of interneuron networks in driving human motor cortex plasticity. Cerebral Cortex 23, 1593-1605 (2013)

20. Hamada, M., Terao, Y., Hanajima, R., Shirota, Y., Nakatani-Enomoto, S., Furubayashi, T., Matsumoto, H., Ugawa, Y.: Bidirectional long-term motor cortical plasticity and metaplasticity induced by quadripulse transcranial magnetic stimulation. Journal of Physiology 586, 3927-3947 (2008)

21. Huang, Y.Z., Edwards, M.J., Rounis, E., Bhatia, K.P., Rothwell, J.: Theta burst stimulation of the human motor cortex. Neuron 45, 201-206 (2005)

22. Ilic, T.V., Meintzschel, F., Cleff, U., Ruge, D., Kessler, K.R., Ziemann, U.: Short-interval paired-pulse inhibition and facilitation of human motor cortex: the dimension of stimulus intensity. Journal of Physiology - London 545, 153-167 (2002)

23. Izhikevich, E.M., Desai, N.S.: Relating STDP to BCM. Neural Computation 15, 1511$1523(2003)$

24. Jirsa, V.K., Haken, H.: A field theory of electromagnetic brain activity. Physical Review Letters 77, 960-963 (1996)

25. Jirsa, V.K., Haken, H.: A derivation of a macroscopic field theory of the brain from the quasi-microscopic neural dynamics. Physica D 99, 503-526 (1997)

26. Kamitani, Y., Bhalodia, V.M., Kubota, Y., Shimojo, S.: A model of magnetic stimulation of neocortical neurons. Neurocomputing 38-40, 697-703 (2001)

27. Liley, D.T.J., Cadusch, P.J., Wright, J.J.: A continuum theory of electro-cortical activity. Neurocomputing 26-27, 795-800 (1999)

28. Loo, C.K., Mitchell, P.B.: A review of the efficacy of transcranial magnetic stimulation (TMS) treatment for depression, and current and future strategies to optimize efficacy. Journal of Affective Disorders 88, 255-267 (2005)

29. Nishiyama, M., Hong, K., Mikoshiba, K., Poo, M., Kato, K.: Calcium stores regulate the polarity and input specificity of synaptic modification. Nature 408, 584-588 (2000)

30. Oberman, L., Edwards, D., Eldaief, M., Pascual-Leone, A.: Safety of theta burst transcranial magnetic stimulation: A systematic review of the literature. Journal of Clinical Neurophysiology 28, 67-74 (2011) 
31. O'Reardon, J.P., Solvason, H.B., Janicak, P.G., Sampson, S., Isenberg, K.E., Nahas, Z., McDonald, W.M., Avery, D., Fitzgerald, P.B., Loo, C., Demitrack, M.A., George, M.S., Sackeim, H.A.: Efficacy and safety of transcranial magnetic stimulation in the acute treatment of major depression: A multisite randomized control trial. Biological Psychiatry 62, 1208-216 (2007)

32. Pell, G.S., Roth, Y., Zangen, A.: Modulation of cortical excitability induced by repetitive transcranial magnetic stimulation: Influence of timing and geometrical parameters and underlying mechanisms. Progress in Neurobiology 93, 59-98 (2011)

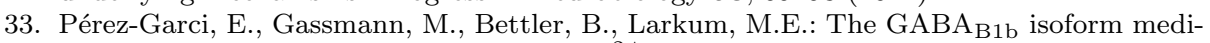
ates long-lasting inhibition of dendritic $\mathrm{Ca}^{2+}$ spikes in layer 5 somatosensory pyramidal neurons. Neuron 50, 603-616 (2006)

34. Pfister, J.P., Gerstner, W.: Triplets of spikes in a model of spike timing-dependent plasticity. Journal of Neuroscience 26, 9763-9682 (2006)

35. Ridding, M.C., Rothwell, J.C.: Is there a future for theraputic use of transcranial magnetic stimulation? Nature Neuroscience 8, 559-567 (2007)

36. Robinson, P.A.: Propagator theory of brain dynamics. Physical Review E 72, 011,904 (2005)

37. Robinson, P.A.: Neural field theory of synaptic plasticity. Journal of Theoretical Biology 285, 156-163 (2011)

38. Robinson, P.A., Rennie, C.J., Rowe, D.L., O‘Connor, S., Gordon, E.: Multiscale brain modelling. Philisophical Transactions of the Royal Society B 360, 1043 (2005)

39. Robinson, P.A., Rennie, C.J., Wright, J.J.: Propagation and stability of waves of electrical activity in the cerebral cortex. Physical Review E 56, 826-840 (1997)

40. Robinson, P.A., Rennie, C.J., Wright, J.J., Bahramali, H., Gordon, E., Rowe, D.L.: Prediction of electroencephalographic spectra from neurophysiology. Physical Review E 63, 021,903 (2001)

41. Roth, B.J., Basser, P.J.: A model of the stimulation of a nerve fiber by electromagnetic induction. IEEE Transactions on Biomedical Engineering 37, 588-597 (1990)

42. Rothkegel, H., Sommer, M., Paulus, W.: Breaks during $5 \mathrm{~Hz}$ rTMS are essential for facilitatory after effects. Clinical Neurophysiology 121, 426-430 (2010)

43. Rothwell, J.: Techniques of transcranial magnetic stimulation. In: S. Boniface, U. Ziemann (eds.) Plasticity in the human nervous system: Investigations with transcranial magnetic stimulation, pp. 26-61. Cambridge University Press (2003)

44. Rubin, J.E., Gerkin, R.C., Bi, G.Q., Chow, C.C.: Calcium time course as a signal for spiking-timing-dependent plasticity. Journal of Neurophysiology 93, 2600-2613 (2005)

45. Sabatini, B.L., Oertner, T.G., Svoboda, K.: The life cycle of $\mathrm{Ca}^{2+}$ ions in dendritic spines. Neuron 33, 439 - 452 (2002)

46. Shah, N.T., Yeung, L.C., Cooper, L.N., Cai, Y., Shouval, H.Z.: A biophysical basis for the inter-spike interaction of spike-timing dependent plasticity. Biological Cybernetics 95, 113-121 (2006)

47. Shouval, H.Z., Bear, M.F., Cooper, L.N.: A unified model of NMDA receptor-dependent bidirectional synaptic plasticity. Proceedings of the National Acadamy of Sciences 99, 10,831-10,836 (2002)

48. Shouval, H.Z., Blais, G.C.S., Blais, B.S., Yeung, L.C., Cooper, L.N.: Converging evidence for a simplified biophysical model of synaptic plasticity. Biological Cybernetics 87, 383-391 (2002)

49. Shouval, H.Z., Kalantzis, G.: Stochastic properties of synaptic transmission affect the shape of spike time-dependent plasticity curves. Journal of Neurophysiology 93, 10671073 (2005)

50. Silva, S., Basser, P.J., Miranda, P.C.: Elucidating the mechanisms and loci of neuronal excitation by transcranial magnetic stimulation using a finite element model of a cortical sulcus. Clinical Neurophysiology 119, 2405-2413 (2008)

51. Steyn-Ross, D.A., Steyn-Ross, M.L., Sleigh, J.W., Wilson, M.T., Gillies, I.P., Wright, J.J.: The sleep cycle modelled as a cortical phase transition. Journal of Biophysics 31, 547-569 (2005)

52. Steyn-Ross, M.L., Steyn-Ross, D.A., Sleigh, J.W., Liley, D.T.J.: Theoretical electroencephalogram stationary spectrum for a white-noise-driven cortex: Evidence for a general anesthetic-induced phase transition. Physical Review E 60, 7299-7311 (1999)

53. Steyn-Ross, M.L., Steyn-Ross, D.A., Wilson, M.T., Sleigh, J.W.: Modeling brain activation patterns for the default and cognitive states. NeuroImage 45, $289-311$ (2009) 
54. Talelli, P., Wallace, A., Dileone, M., Hoad, D., Cheeran, B., Oliver, R., VandenBos, M., Hammerbeck, U., Barratt, K., Gillini, C., Musumeci, G., Boudrias, H.H., Cloud, G.C., Ball, J., Marsden, J.F., Ward, N.S., Lazzaro, V.D., Greenwood, R.G., Rothwell, J.C.: Theta burst stimulation in the rehabilitation of the upper limb: A semirandomized, placebocontrolled trial in chronic stroke patients. Neurorehabilitation and Neural Repair 26, 976-987 (2012)

55. Thut, G., Pascual-Leone, A.: A review of combined TMS-EEG studies to characterize lasting effects of repetitive TMS and assess their usefulness in cognitive and clinical neuroscience. Brain Topography 22, 219-232 (2010)

56. Tsutsumi, R., Hanajima, R., Terao, Y., Shirota, Y., Ohminami, S., Shimizu, T., Tanaka, N., Ugawa, Y.: Effects of the motor cortical quadripulse transcranial magnetic stimulation (QPS) on the contralateral motor cortex and interhemispheric interactions. Journal of Neurophysiology 111, 26-35 (2014)

57. Vahabzadeh-Hagh, A.M., Muller, P.A., Gersner, R., Zangen, A., Rotenberg, A.: Translational neuromodulation: approximating human transcranial magnetic stimulation protocols in rats. Neuromodulation 15, 296-305 (2012)

58. Walsh, V., Cowey, A.: Transcranial magnetic stimulation and cognitive neuroscience. Nature Reviews: Neuroscience 1, 73-79 (2000)

59. Wang, H.X., Gerkin, R.C., Nauen, D.W., Bi, G.Q.: Coactivation and timing-dependent integration of synaptic potentiation and depression. Nature Neuroscience 8, 187-193 (2005)

60. Wilson, M.T., Goodwin, D.P., Brownjohn, P.W., Shemmell, J., Reynolds, J.N.J.: Numerical modelling of plasticity induced by transcranial magnetic stimulation. Journal of Computational Neuroscience 36, 499-514 (2014)

61. Wilson, M.T., Robinson, P.A., O'Neill, B., Steyn-Ross, D.A.: Complementarity of spikeand rate-based dynamics of neural systems. PLoS Computational Biology 8, e1002,560 (2012)

62. Wilson, M.T., Sleigh, J.W., Steyn-Ross, D.A., Steyn-Ross, M.L.: General anestheticinduced seizures can be explained by a mean-field model of cortical dynamics. Anesthesiology 104, 588-593 (2006)

63. Wittenberg, G.M., Wang, S.S.H.: Malleability of spike-timing-dependent plasticity at the CA3-CA1 synapse. Journal of Neuroscience 26, 6610-6617 (2006) 